\title{
Driving term experiments at CERN
}

\author{
M. Benedikt, F. Schmidt, R. Tomás, and P. Urschütz \\ CERN, Geneva, Switzerland
}

\section{A. Faus-Golfe}

Instituto de Fisica Corpuscular, Valencia, Spain

(Received 29 November 2006; published 22 March 2007)

\begin{abstract}
Driving Term experiments have been performed both at small [PS (Proton Synchrotron) Booster] and large accelerators [SPS (Super Proton Synchrotron)] at CERN. The theory of how to measure driving terms is reviewed. A wealth of SPS experiments is shown together with a successful comparison with model calculations. The PS Booster studies aimed at optimizing the machine performance by measuring and correcting selected driving terms.
\end{abstract}

DOI: 10.1103/PhysRevSTAB.10.034002

PACS numbers: 41.85. $-\mathrm{p}, 29.27 .-\mathrm{a}, 05.45 .-\mathrm{a}$

\section{INTRODUCTION}

Driving term experiments had first been applied in 1988 at the LEAR machine at CERN [1]. In 1998, the technique was firmly connected to the Normal Form theory [2,3]. Using this modified approach, the first pioneering experiments were performed at the SPS and LEP in the years 1996 until 1999 [4].

From 1999 until 2003, extensive studies have been performed at the SPS using strong sextupoles to drive nonlinear resonances. In the first part of this report, the theory is outlined showing how the longitudinally varying driving terms can be measured. It will also be demonstrated that the theory must be modified if realistic particle distributions are considered rather than single particles.

In the second part, the most relevant results of the SPS experiments will be summarized. In particular, it will be demonstrated that one can measure nonlinear resonances around the accelerator and that this information can be used to detect polarity errors in the powering of sextupoles or to find missing sextupoles. It will also be shown that the linear coupling resonance can be measured and corrected with high precision.

In the third part of this report, we will cover the driving term experiments at the CERN PS Booster performed between 2002 and 2004. These studies rely on the same measuring technique. However, the emphasis for these machine studies has been to demonstrate that one can build up rather easily a 1000 turn beam position monitor (BPM) system from scratch, measure with high precision resonance driving terms under difficult experimental conditions (e.g. no kicker, partial chromaticity correction only), and use that information for an optimization of the machine performance.

\section{SPECTRAL RESPONSE TO PARTICLE DISTRIBUTIONS}

This section studies how the beam decoherence affects the spectrum of the turn-by-turn centroid motion. It is described how the different spectral lines are differently altered when decoherence processes take place. This is a fundamental ingredient in the measurement of resonance terms since amplitude detuning is almost unavoidable in the presence of nonlinear fields.

\section{A. Analytical approach}

In [3] the relation between the spectral lines of the single particle motion and the resonance driving terms was derived as

$$
\begin{aligned}
h_{x}^{-}(N)= & {\sqrt{2 I_{x}}}^{i\left(2 \pi \nu_{x} N+\psi_{x_{0}}\right)}-2 i \\
& \times \sum_{j k l m} j f_{j k l m}\left(2 I_{x}\right)^{(j+k-1) / 2}\left(2 I_{y}\right)^{(l+m) / 2} \\
& \times e^{i\left[(1-j+k)\left(2 \pi \nu_{x} N+\psi_{x_{0}}\right)+(m-l)\left(2 \pi \nu_{y} N+\psi_{y_{0}}\right)\right]},
\end{aligned}
$$

where $h_{x}^{-}(N)$ is the turn-by-turn complex horizontal variable, $I_{x, y}$ are the action variables, $\nu_{x, y}$ are the transverse tunes, $\psi_{x_{0}, y_{0}}$ are the initial phases, and $f_{j k l m}$ are the generating function terms. In a real machine BPMs are used to record the turn-by-turn transverse position of the centroid of the beam. Therefore the relation between the spectral lines of the motion of the centroid and the resonance driving terms has to be found. It is assumed that the coordinates of the centroid are given by averaging over a Gaussian distribution of noninteracting particles. The most important process that affects the centroid motion is the decoherence introduced by the amplitude detuning and chromaticity.

The amplitude detuning is due to the presence of nonlinear magnetic fields. The tunes of the particle also depend on its momentum deviation. The parameter used to quantify this dependence is the chromaticity $Q^{\prime}$, defined as the derivative of the tune versus the relative momentum deviation. In addition, the energy of off-momentum particles performs harmonic oscillations around the reference energy due to the effect of the radio-frequency (RF) cavities. The frequency of these oscillations is the synchrotron tune 
$Q_{s}$. In the following, the spectrum of the motion of the centroid of the beam is studied assuming Gaussian particle distributions.

\section{Tune dependence}

Nonlinear elements introduce a dependence of the tunes $\nu_{x}$ and $\nu_{y}$ on the horizontal and vertical invariants $I_{x}$ and $I_{y}$. The linear approximation is given by

$$
\begin{aligned}
& \nu_{x}=Q_{x}+\nu_{x x}^{\prime} 2 I_{x}+\nu_{x y}^{\prime} 2 I_{y} \\
& \nu_{y}=Q_{y}+\nu_{y x}^{\prime} 2 I_{x}+\nu_{y y}^{\prime} 2 I_{y},
\end{aligned}
$$

where the $\nu^{\prime}$ are the amplitude detuning terms.

Off-momentum particles will experience an additional tune oscillation given by the expression [5]

$$
\Delta \nu_{x, y}=\frac{Q_{x, y}^{\prime} \delta}{\pi Q_{s} N} \cos \left(\pi Q_{s} N+\psi_{s}\right) \sin \left(\pi Q_{s} N\right),
$$

where $Q_{s}$ is the longitudinal or synchrotron tune, $\psi_{s}$ is the initial phase, and $\delta$ is the momentum deviation. $Q_{x}^{\prime}$ and $Q_{y}^{\prime}$ are the horizontal and vertical chromaticities and $N$ is the turn number.

\section{The centroid motion and its spectrum}

The centroid position and momentum are computed by averaging over the bunch distribution. The turn-by-turn motion of each particle $h_{x}^{-}(N)$ is given by Eq. (1) as a function of its transverse amplitudes and initial phases. Amplitude detuning and chromaticity are taken into account by replacing the tunes with Eqs. (2) and (3). At every turn $N$, the centroid normalized horizontal coordinate is given by the following expression:

$$
\begin{aligned}
\overline{h_{x}^{-}}(N)= & \int_{0}^{\infty} d I_{x} \int_{0}^{\infty} d I_{y} \int_{0}^{\infty} d \delta \int_{0}^{2 \pi} d \psi_{x} \int_{0}^{2 \pi} d \psi_{y} \\
& \times \int_{0}^{2 \pi} d \psi_{s} \rho_{x}\left(I_{x}, \psi_{x}\right) \rho_{y}\left(I_{y}, \psi_{y}\right) \rho_{s}\left(\delta, \psi_{s}\right) h_{x}^{-}(N) .
\end{aligned}
$$

This integral involves the six variables of the phase space and $h_{x}^{-}(N)$ is given by Eq. (1) including amplitude detuning and chromaticity. In the Appendix, it is describes how to solve four of these integrals. It does not seem possible to integrate over $I_{x}$ and $I_{y}$. However, we are more interested in the Fourier transformation of the centroid turn-by-turn motion, $\overline{h_{x}^{-}}(N)$, represented by $H_{x}^{-}(w)$. Since the Fourier transformation is a linear operator, each term of the righthand side (rhs) of Eq. (1) gives a contribution to $H_{x}^{-}(w)$ which is expressed by

$$
\begin{aligned}
H_{x}^{-}(w) & =\int d N \overline{h_{x}^{-}}(N) e^{-i w N} \\
& \equiv H_{x, \text { tune }}^{-}(w)+\sum_{j k l m} H_{x, j k l m}^{-}(w) .
\end{aligned}
$$

The steps to solve this integral are described in the Appendix. By solving this integral, a Dirac delta function permits to integrate over $I_{x}$. Nevertheless it is not possible to solve analytically the integral over $I_{y}$. The different terms of the rhs of Eq. (4) are reexpressed as

$$
\begin{aligned}
H_{x, \text { tune }}^{-}(w)= & \mathcal{A}_{x, \text { tune }}^{-}(w) e^{-\gamma_{10}^{2}} \mathrm{I}_{0}\left(\gamma_{10}^{2}\right) \\
& +\sum_{q=1}^{\infty} e^{-\gamma_{10}^{2}} \mathrm{I}_{q}\left(\gamma_{10}^{2}\right)\left[\mathcal{A}_{x, \text { tune }}^{-}\left(w+q 2 \pi Q_{s}\right)\right. \\
& \left.+\mathcal{A}_{x, \text { tune }}^{-}\left(w-q 2 \pi Q_{s}\right)\right] \\
H_{x, j k l m}^{-}(w)= & \mathcal{A}_{x, j k l m}^{-}(w) e^{-\gamma_{(1-j+k)(m-l)}^{2}} \mathrm{I}_{0}\left(\gamma_{(1-j+k)(m-l)}^{2}\right) \\
& +\sum_{q=1}^{\infty} e^{-\gamma_{(1-j+k)(m-l)}^{2}} \mathrm{I}_{q}\left(\gamma_{(1-j+k)(m-l)}^{2}\right) \\
& \times\left[\mathcal{A}_{x, j k l m}^{-}\left(w+q 2 \pi Q_{s}\right)\right. \\
& \left.+\mathcal{A}_{x, j k l m}^{-}\left(w-q 2 \pi Q_{s}\right)\right],
\end{aligned}
$$

where the functions $\mathcal{A}_{x \text {,tune }}^{-}(w)$ and $\mathcal{A}_{x, j k l m}^{-}(w)$ are defined in Eqs. (A17) and (A18). They still contain an integral over the coordinate $I_{y}$ and they are single peak distributions with their maxima close to the frequencies $\nu_{x}$ and $(1-j+$ $k) \nu_{x}+(m-l) \nu_{y}$, respectively. Their amplitudes contain the generating function terms and the reduction factor $\left|(1-j+k) \nu_{x x}^{\prime}+(m-l) \nu_{y x}^{\prime}\right|$. These factors are due to the decoherence and increase the difficulty to measure the resonance terms since they reduce the signal of interest. The functions $H_{x, \text { tune }}^{-}(w)$ and $H_{x, j k l m}^{-}(w)$ are equal to $\mathcal{A}_{x, \text { tune }}^{-}(w)$ and $\mathcal{A}_{x, j k l m}^{-}(w)$ when the chromaticity is zero. From those expressions, one can read that the effect of chromaticity is to add an infinite number of sidebands at $\pm q 2 \pi Q_{s}$ to every line. The shape of the sidebands is the same as the one of the main peak and their amplitudes decrease as $q$ increases. Simpler expressions are found when considering only one transverse dimension or when looking at special spectral lines.

One transverse dimension. - When only one of the transverse planes is relevant analytical expressions of the functions $\mathcal{A}_{x, \text { tune }}^{-}(w)$ and $\mathcal{A}_{x, j k l m}^{-}(w)$ can be achieved. Considering only the horizontal plane, the integrals over $I_{y}$ from Eqs. (A17) and (A18) are dropped and $I_{y}$ is set to zero, resulting in

$$
\begin{aligned}
\mathcal{A}_{x, \text { tune }}^{-}(w)= & \frac{1}{\left|\nu_{x x}^{\prime}\right|} \sqrt{2 I_{x}(w)} e^{-(1 / 2)\left[2 I_{x}(w)+\bar{A}_{x}^{2}\right]} \\
& \times \mathrm{I}_{1}\left[\bar{A}_{x} \sqrt{2 I_{x}(w)}\right] \\
\mathcal{A}_{x, j k 00}^{-}(w)= & -\frac{2 i j f_{j k 00}}{\left|(1-j+k) \nu_{x x}^{\prime}\right|} \\
& \times\left[2 I_{x}(w)\right]^{(j+k-1) / 2} e^{-(1 / 2)\left[2 I_{x}\left(w, I_{y}\right)+\bar{A}_{x}^{2}\right]} \\
& \times \mathrm{I}_{(1-j+k)}\left[\bar{A}_{x} \sqrt{2 I_{x}(w)}\right]
\end{aligned}
$$




$$
2 I_{x}(w)=\frac{1}{(1-j+k) \nu_{x x}^{\prime}}\left[w / 2 \pi-(1-j+k) Q_{x}\right] .
$$

Therefore $H_{x}^{-}(w)$ is given by introducing these expressions into Eqs. (5) and (6). To compare the former $\mathcal{A}_{x, j k 00}^{-}$to the single particle case, the Fourier spectrum of the motion of a single particle is given with a similar notation [see Eq. (1)]:

$$
\begin{aligned}
h_{x, \text { tune }}^{-}(w)= & \bar{A}_{x} \delta_{\text {Dirac }}\left(w / 2 \pi-\nu_{x}\right) \\
h_{x, j k 00}^{-}(w)= & -2 i j f_{j k 00}\left(\bar{A}_{x}\right)^{(j+k-1) / 2} \\
& \times \delta_{\text {Dirac }}\left[w / 2 \pi-(1-j+k) \nu_{x}\right],
\end{aligned}
$$

where $\delta_{\text {Dirac }}$ is the Dirac delta function and the oscillation amplitude of the single particle is $\sqrt{2 I_{x}}=\bar{A}_{x}$. The most relevant difference between the single particle and the multiparticle cases is that the spectral lines are no longer spikes but distributions scaled down by the factor $\mid(1-$ $j+k) \nu_{x x}^{\prime} \mid$. These factors are called decoherence factors and depend only on the frequency of the spectral line and on the amplitude detuning coefficients. Throughout this paper, the spectrum of experimental data is normalized to the fundamental line (tune line) to compare to model data more easily. This normalization also takes care of errors in the calibration of the BPMs. The decoherence factor corresponding to the horizontal spectral line $(m, n)$ is given by

$$
\left|m+n \frac{\nu_{y x}^{\prime}}{\nu_{x x}^{\prime}}\right|
$$

In particular, for the one-dimensional case the decoherence factor corresponding to the spectral line $(m, 0)$ is $|m|$, e.g., the spectral line of the $(3,0)$ resonance is scaled down by a factor of 2 .

The zero frequency line.-The generating term $f_{j k l m}$ corresponds to a zero frequency line in the horizontal plane when

$$
1-j+k=0 \quad \text { and } \quad m-l=0 .
$$

Any term of the form $H_{x, j(j-1) m m}^{-}$for arbitrary $j$ and $m$ fulfills the previous relations and therefore corresponds to the zero frequency line. Note that these terms are not distributions since they are only defined at $w=0$. Starting from Eq. (A4) and evaluating the integrals over the transverse phases, one obtains

$$
\begin{aligned}
H_{x, j(j-1) m m}^{-}= & -2 i j f_{j(j-1) m m} \int_{0}^{\infty} d I_{x} \int_{0}^{\infty} d I_{y}\left(2 I_{x}\right)^{(j-1)}\left(2 I_{y}\right)^{m} \\
& \times e^{-(1 / 2)\left(2 I_{x}+\bar{A}_{x}^{2}+2 I_{y}+\bar{A}_{y}^{2}\right)} I_{0}\left(\bar{A}_{x} \sqrt{2 I_{x}}\right) I_{0}\left(\bar{A}_{y} \sqrt{2 I_{y}}\right) .
\end{aligned}
$$

This integral can be computed for all $j>0$ and $m \geq 0$ leading to hypergeometric functions. For $m=0$ these hypergeometric functions are simply polynomials of order $2(j-1)$ in $\bar{A}_{x}$. The cases $H_{x, 2100}^{-}$and $H_{x, 3200}^{-}$are related to sextupolar and decapolar fields, respectively, and they are given by

$$
\begin{aligned}
& H_{x, 2100}^{-}=-4 i f_{2100}\left(2+\bar{A}_{x}^{2}\right) \\
& H_{x, 3200}^{-}=-6 i f_{3200}\left(8+8 \bar{A}_{x}^{2}+\bar{A}_{x}^{4}\right) .
\end{aligned}
$$

Note that the spectral lines are nonzero even for $\bar{A}_{x}=0$. In the single particle case only the highest order term of Eq. (14) exists:

$$
h_{x, 2100}^{-}=-4 i f_{2100} \bar{A}_{x}^{2} \quad h_{x, 3200}^{-}=-6 i f_{3200} \bar{A}_{x}^{4} .
$$

For large values of $\bar{A}_{x}$, Eqs. (14) and (15) are approximately the same and there is no decoherence factor.

\section{B. Simulations}

In this section some of the analytical expressions derived in Sec. II A are compared to computer simulations. A computer model of the super proton synchrotron (SPS) has been used for the tracking of a large number of particles corresponding to an off-center Gaussian distribution in the horizontal phase space (SixTrac [6]). The turn-by-turn position of the centroid is given by the average position of the particles. The Fourier transform of the centroid data was computed with SUSSIX [7]. Amplitude detuning has been introduced by eight extraction sextupoles. Half of the sextupoles are powered to a positive value while the other half has negative strength. All sextupoles are placed at optically equivalent positions. Various kick amplitudes ranging from $1 \sigma$ to $6 \sigma$ have been simulated. As an illustration, the simulation data for the case with a kick of $4 \sigma$ is shown in Fig. 1. The amplitude detuning leads to a damping of the oscillations. Its Fourier spectrum is shown in Fig. 2. The labels $(m, n)$ mean that the frequency of the corresponding line is $m \nu_{x}+n \nu_{y}$. The tune line is the $(1,0)$ line and its amplitude is used to normalize the spectrum. The lowest order horizontal spectral lines arising from the sextupoles are $(-2,0),(2,0)$, and $(0,0)$ which are propor-

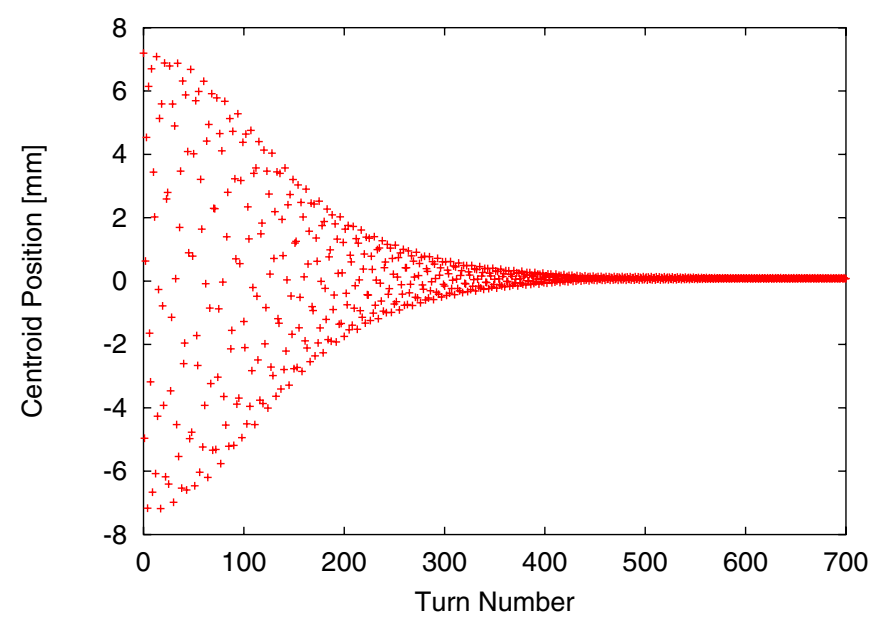

FIG. 1. (Color) Horizontal position of the centroid of the beam versus turn number for the SPS with extraction sextupoles on. 


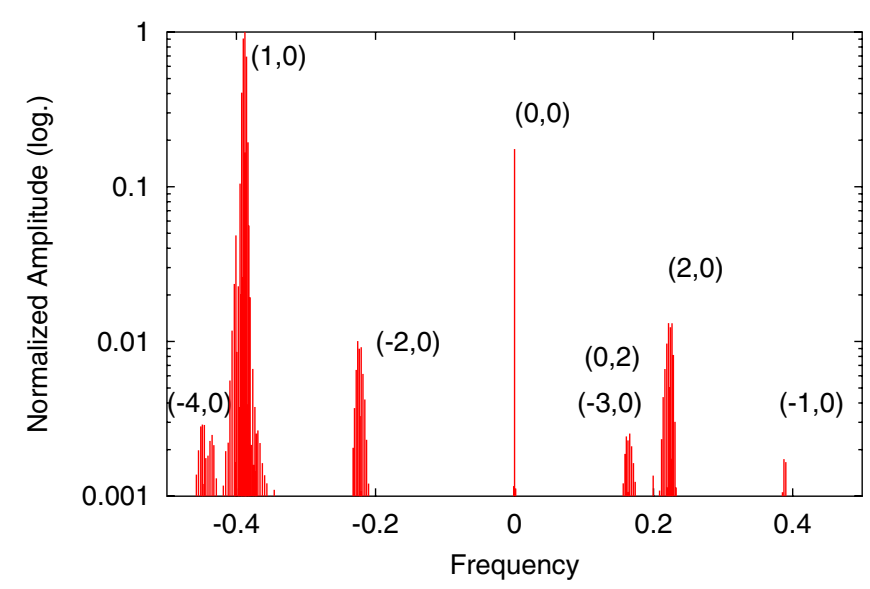

FIG. 2. (Color) FFT spectrum of the horizontal centroid motion of the beam for the SPS with extraction sextupoles on.

tional to the generating terms $f_{3000}, f_{1200}$, and $f_{2100}$, respectively. Higher order terms have been ignored although we have verified that they tend to be very small. When particle distributions are considered, the spectral lines become wide distributions instead of spikes, with the exception of the $(0,0)$ line (Sec. II A 2).

In Sec. II A 2 the expressions for the Fourier spectrum for one transverse dimension are given. The analytical expressions describing the tune line $(1,0)$ are given by

$$
\mathcal{H}_{x, \text { tune }}^{-}(w)=\frac{1}{\left|\nu_{x x}^{\prime}\right|} \sqrt{2 I_{x}(w)} e^{-(1 / 2)\left[2 I_{x}(w)+\bar{A}_{x}^{2}\right]} \mathrm{I}_{1}\left(\bar{A}_{x} \sqrt{2 I_{x}(w)}\right)
$$

$$
2 I_{x}(w)=\frac{1}{\nu_{x x}^{\prime}}\left(w / 2 \pi-Q_{x}\right)
$$

The parameters needed for the evaluation of this expression are taken from the single particle tracking. These parameters are the horizontal tune for zero oscillation amplitude $\left(Q_{x}\right)$ and the horizontal amplitude detuning $\left(\nu_{x x}^{\prime}\right)$. In Fig. 3 the tune line obtained from the Fourier transform of the simulation with the particle distribution is compared to the analytical model. The agreement between the two curves is sufficiently good to suggest that tune measurements might be improved by fitting the predicted curve to the Fourier spectrum of the measured data.

The distribution of the line $(-2,0)$ is the term $H_{x, 3000}(w)$ which is obtained from Eq. (8),

$$
\begin{aligned}
& \mathcal{H}_{x, 3000}^{-}(w)=-\frac{6 i f_{3000}}{\left|2 \nu_{x x}^{\prime}\right|} 2 I_{x}(w) e^{-(1 / 2)\left[2 I_{x}\left(w, I_{y}\right)+\bar{A}_{x}^{2}\right]} \\
& \times \mathrm{I}_{2}\left[\bar{A}_{x} \sqrt{2 I_{x}(w)}\right] \\
& 2 I_{x}(w)=\frac{1}{-2 \nu_{x x}^{\prime}}\left(w / 2 \pi+2 Q_{x}\right) .
\end{aligned}
$$

The parameters needed for the evaluation of these expres-

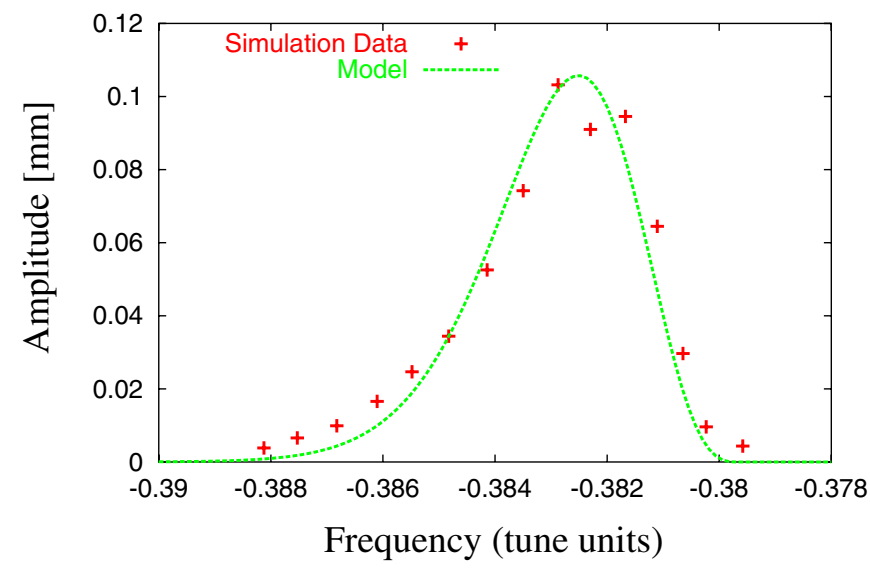

FIG. 3. (Color) Distribution of the tune peak for model and simulation versus frequency (in tune units) for the SPS with extraction sextupoles on.

sions are the horizontal tune $\left(Q_{x}\right)$, the amplitude detuning $\left(\nu_{x x}^{\prime}\right)$, and the generating term $f_{3000}$. The first two were already obtained earlier. The generating term was calculated analytically. In Fig. 4 the line $(-2,0)$ obtained from the Fourier transform of the centroid simulation data is compared to the analytical prediction. The frequency of the peak of the curve and its width are well predicted although there is a small discrepancy in the right tail. It remains unclear whether this discrepancy comes from the simulation or from the decoherence model.

The line $(0,0)$ has zero width and its amplitude as a function of the kick is given by $H_{x, 2100}^{-}$, Eq. (15). This amplitude only depends on the generating term $f_{2100}$. The amplitude of the line $(0,0)$ is shown in Fig. 5 versus kick amplitude. It has been computed with the same procedures as the line $(-2,0)$ and it is compared to the prediction of Eq. (15). Both agree almost perfectly. As predicted this line exists even without a kick.

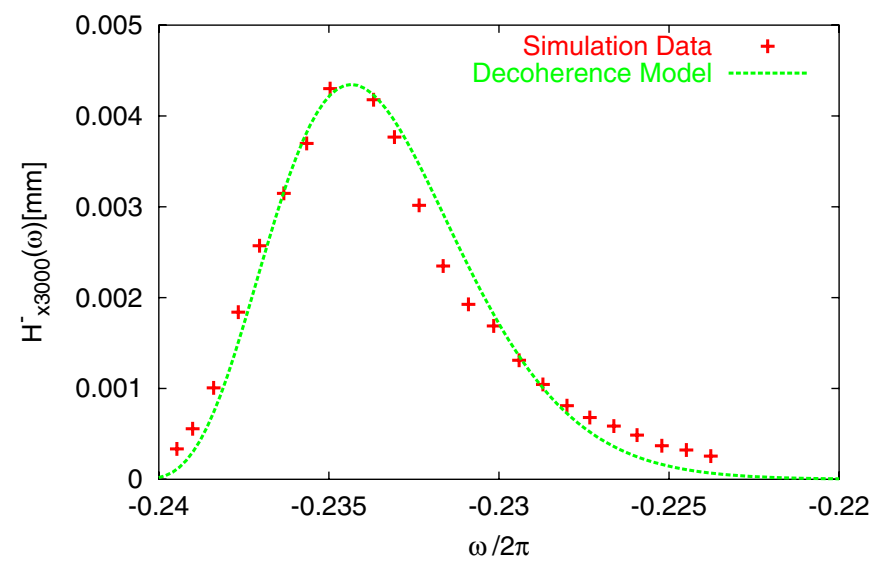

FIG. 4. (Color) Distribution of the line $(-2,0)$ for model and simulation against frequency for the SPS with extraction sextupoles on. 


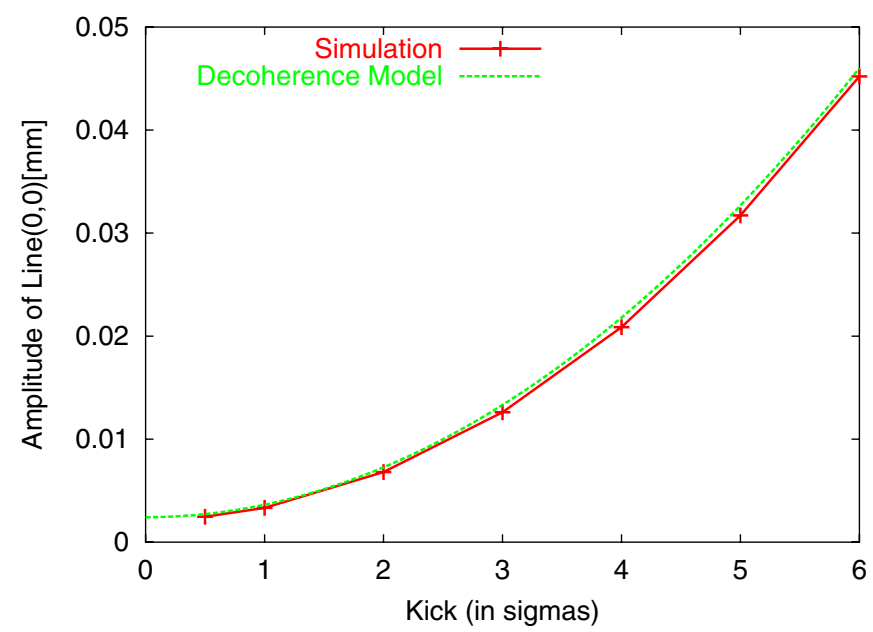

FIG. 5. (Color) Amplitude of the line $(0,0)$ for model and simulation versus the kick $\overline{A_{x}}$ in sigmas for the SPS with extraction sextupoles on.

\section{LOCALIZATION OF MULTIPOLES}

The longitudinal variation around the ring of the Hamiltonian and the generating function terms are studied in this section. It is shown how measuring the amplitude of the different resonance driving terms along the ring allows the identification of the longitudinal positions of multipolar kicks. Therefore it is possible to identify field errors and their location. This method is of great interest for commissioning and operating an accelerator.

\section{A. Longitudinal variation of resonance terms}

The dependence of the one turn map on the longitudinal coordinate is studied by constructing two maps starting at two different locations $\left(s_{1}\right.$ and $s_{2}$ ), separated by a linear section $M_{1}$ and a nonlinear multipole kick $e^{: h_{1}:}$, as represented in Fig. 6. The corresponding one turn maps are expressed as [8]

$$
\begin{aligned}
& \mathcal{M}^{(1)}=M_{N+1} e^{: h_{N}:} M_{N} e^{: h_{N-1}: \cdots} e^{: h_{2}:} M_{2} e^{: h_{1}:} M_{1} \text {, }
\end{aligned}
$$

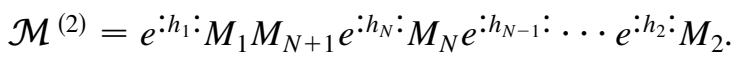

Both maps can be expressed in their respective normalized phase space as follows:

$$
\begin{aligned}
& \mathcal{M}^{(1)}=e^{: h^{(1)}}: R, \\
& \mathcal{M}^{(2)}=e^{: h^{(2)}}: R,
\end{aligned}
$$

where $R$ represents the linear one turn map in the normalized coordinates. The quantities $h^{(1)}$ and $h^{(2)}$ are given by [8]

$$
\begin{aligned}
h^{(1)}= & A_{1} M_{1} h_{1}+A_{1} M_{1} M_{2} h_{2}+\cdots \\
& +A_{1} M_{1} M_{2} \cdots M_{N} h_{N} \\
h^{(2)}= & A_{2} M_{2} h_{2}+A_{2} M_{2} M_{3} h_{3}+\cdots \\
& +A_{2} M_{2} \cdots M_{N+1} M_{1} h_{1},
\end{aligned}
$$

where $A_{1}$ and $A_{2}$ are transformations that bring the local coordinates into the normalized coordinates. Applying $A_{2} M_{1}^{-1} A_{1}^{-1}$ to $h^{(1)}$, the following relation holds between $h^{(1)}$ and $h^{(2)}$ :

$$
h^{(2)}=A_{2} M_{1}^{-1} A_{1}^{-1} h^{(1)}-A_{2} h_{1}+A_{2} M_{2} \cdots M_{N+1} M_{1} h_{1} .
$$

Using $R=A_{2} M_{2} \ldots M_{N+1} M_{1} A_{2}^{-1}$ and grouping terms with $h_{1}$ yields

$$
h^{(2)}=A_{2} M_{1}^{-1} A_{1}^{-1} h^{(1)}+(R-1) A_{2} h_{1},
$$

which expresses the relation between the two Hamiltonians. The generating functions at both locations are defined as

$$
F^{(1)}=\frac{1}{1-R} h^{(1)}
$$
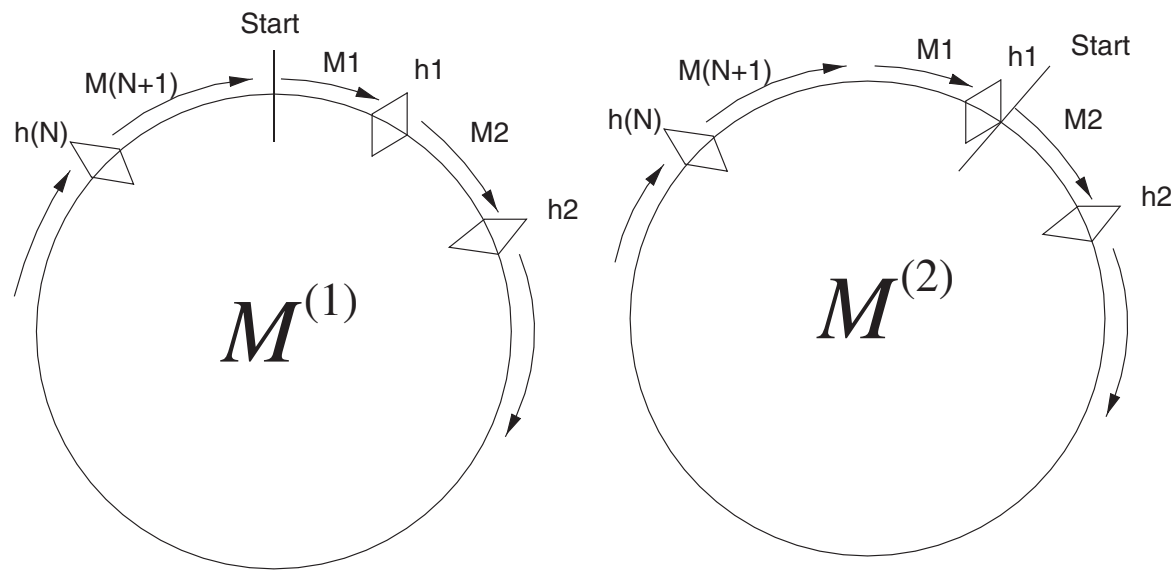

FIG. 6. Schematic view of the ring for maps at two different longitudinal starting locations. The arrows represent the linear maps and the diamonds the nonlinear multipolar kicks. 


$$
F^{(2)}=\frac{1}{1-R} h^{(2)}
$$

By introducing $h^{(2)}$ from Eq. (26) into Eq. (28) and using the fact that $R$ commutes with $A_{2} M_{1}^{-1} A_{1}^{-1}$, since they are both rotations in the normalized phase space, the relation between $F^{(2)}$ and $F^{(1)}$ is expressed as

$$
F^{(2)}=A_{2} M_{1}^{-1} A_{1}^{-1} F^{(1)}-A_{2} h_{1} .
$$

This equation shows how the generating function changes in a section with a nonlinear element. If there are no nonlinear kicks between the two initial longitudinal positions, the term $A_{2} h_{1}$ of Eq. (29) will vanish and $F^{(1)}$ and $F^{(2)}$ will be smoothly related by the change of phase given by $A_{2} M_{1}^{-1} A_{1}^{-1}$. Otherwise, when there is a nonlinear kick in between, represented by $h_{1}$, an abrupt change in the phase and the amplitude of the corresponding generating term can be expected.

To illustrate the usefulness of this technique, a single particle simulation is done using a realistic model of the SPS with the extraction sextupoles. Apart from the extraction sextupoles, the SPS has a large number of chromaticity sextupoles used to correct the chromaticity in normal operation. The code SixTrac is used to track a single particle through the lattice. Turn-by-turn data is obtained at many longitudinal locations of the ring and the amplitude of the spectral line $(-2,0)$, proportional to $f_{3000}$, is obtained from the fast-Fourier-transform (FFT) of the data. The results are shown in Fig. 7. The location of the extraction sextupoles is marked with vertical lines. The largest abrupt changes of the curve occur at the location of extraction sextupoles, since these are the strongest sextu-

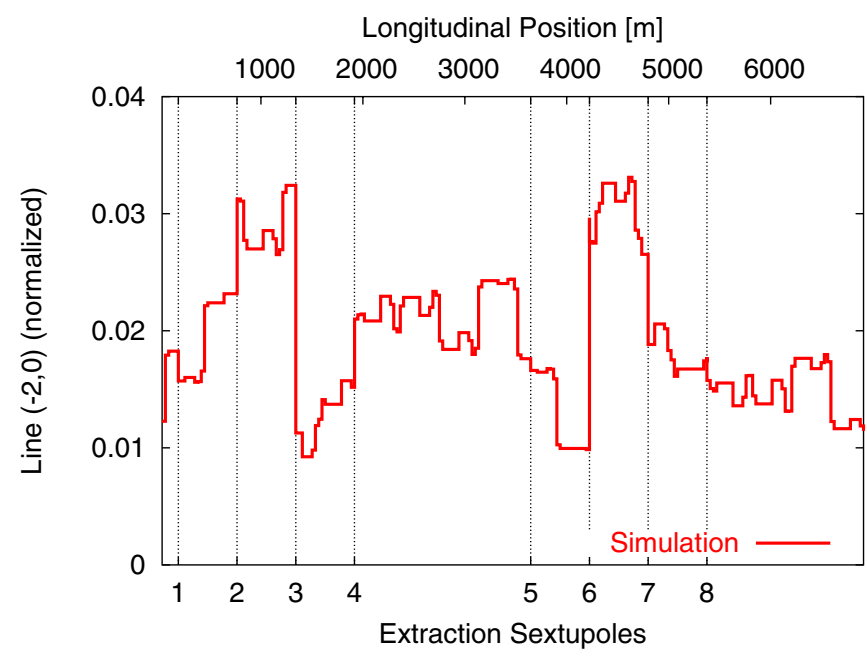

FIG. 7. (Color) Simulated amplitude of the line $(-2,0)$ versus the longitudinal coordinate for the nominal SPS model with extraction sextupoles with polarities: $(++++----30)$ A. The vertical lines indicate the location of the extraction sextupoles. poles. However, there are smaller jumps all around the ring due to the chromaticity sextupoles.

\section{SPS EXPERIMENTS}

The SPS is the largest accelerator in the LHC injector chain. It receives $26 \mathrm{GeV}$ protons from the PS and accelerates the beam up to $450 \mathrm{GeV}$, the injection energy of the LHC.

A series of experiments to measure resonance driving terms in the SPS has been performed between 2000 and 2002 . Prior to this study pioneering experiments were carried out $[9,10]$. The extraction sextupoles were used to create a controllable amount of nonlinearities. Furthermore the skew quadrupoles were also used to create and to correct coupling.

\section{A. SPS instrumentation}

The SPS is equipped with a large collection of instruments used to control the beam and measure its parameters. In the following a brief description of the instruments used in the experiments is given.

BPM and MOPOS system. - The majority of the BPMs of the SPS are of electrostatic type. The SPS is equipped with about 110 BPMs in either transverse plane and they are uniformly distributed around the $7 \mathrm{~km}$ circumference of the ring. The phase advance between consecutive pickups is almost $\pi / 2$. The signal coming out of the BPMs is electronically processed and 1000 turns per BPM are collected by the multiorbit position system (MOPOS) system. In order to obtain a reliable BPM signal during the experiments the timing parameter and the gain of the electronics had to be carefully adjusted.

$Q$-kickers. - The SPS is equipped with a horizontal and a vertical $Q$-kicker. They are called $Q$-kickers since in normal operation they are used to measure the transverse tunes. In our experiments these kickers are used to excite the transverse motion for energies of $80 \mathrm{GeV}$ or below.

Extraction kicker.-This horizontal kicker is more powerful than the $Q$-kickers. In normal operation it is used for the fast beam extraction. In our experiments at $120 \mathrm{GeV}$ this kicker was used to excite the horizontal betatron motion.

Extraction sextupoles. - In normal operation these sextupoles are used for the slow or resonant extraction of the beam. The SPS is equipped with eight extraction sextupoles. In our experiments they were used to create a controllable amount of nonlinearity.

Skew quadrupoles. - The SPS is equipped with six skew quadrupoles powered in series. In normal operation they are used to compensate the coupling errors of the machine.

\section{B. Basic measurements}

The basic procedure of the resonance driving terms experiments is to acquire various sets of BPM data, from 


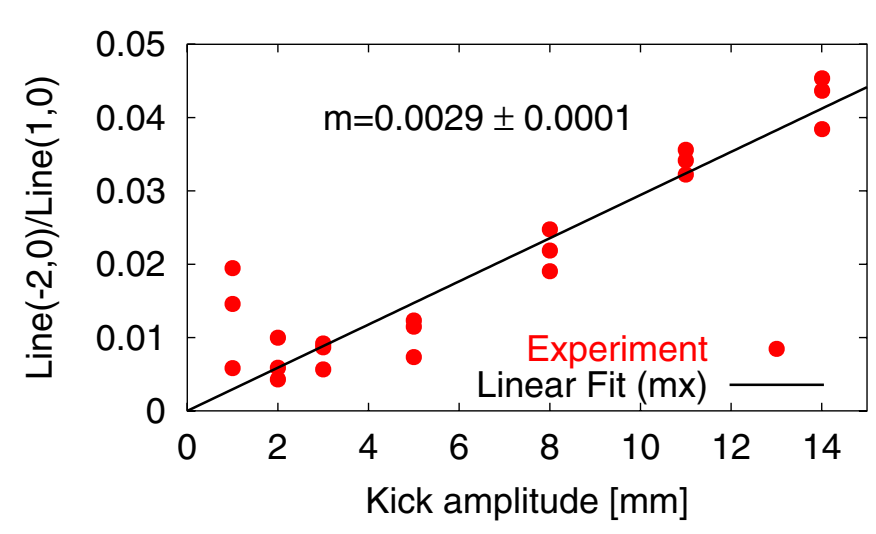

FIG. 8. (Color) Normalized amplitude of the spectral line $(-2,0)$ versus horizontal kick amplitude for one pickup.

all the available BPMs, for different oscillation amplitudes. The complex signal constructed from two adjacent pickups is Fourier analyzed by using the SUSSIX [7] code. For every pickup the normalized amplitudes of the sextupolar spectral lines are plotted versus kick strength and a line is fitted through the data with the additional constraint to go through the origin. As an illustration the spectral line $(-2,0)$ for a particular pickup is plotted against the kick amplitude (Fig. 8). Three measurements for every kick strength were done to assess random errors. The slope of the fitted line is related to the corresponding resonance term [see Eq. (1)]:

$$
\begin{aligned}
& \left|f_{3000}\right|=\frac{1}{6}\left(\frac{m_{(-2,0)}}{c_{k}}\right)\left[\mu \mathrm{m}^{-1 / 2}\right] \\
& \left|f_{1200}\right|=\frac{1}{2}\left(\frac{m_{(2,0)}}{c_{k}}\right)\left[\mu \mathrm{m}^{-1 / 2}\right]
\end{aligned}
$$

where the $m$ is the measured slope and its subscript denotes the spectral line. The factor $c_{k}$ comes from the calibration of the horizontal kicker. These relations hold as far as the beam does not experience any decoherence. When the centroid oscillations are completely damped due to decoherence caused by amplitude detuning, the spectral lines $( \pm 2,0)$ are reduced by a decoherence factor of 2 . The measurement of a sextupolar resonance term around the ring is compared to the model (Fig. 9). Beam conditions are found in Table I. The level of agreement is good and the variations around the ring are due to the chromaticity sextupoles.

The quality of the data also allows a measurement of the phase of the spectral line $(-2,0)$. From Eq. (1) the amplitude and phase of the spectral line $(1-j+k, m-l)$ are given by the following complex quantity:

$$
-2 i j f_{j k l m}\left(2 I_{x}\right)^{(j+k-1) / 2}\left(2 I_{y}\right)^{(l+m) / 2} e^{i\left[(1-j+k) \psi_{x_{0}}+(m-l) \psi_{y_{0}}\right]} .
$$

The term $f_{j k l m}$ is proportional to the sum over all nonlinear elements of the same type, i.e.

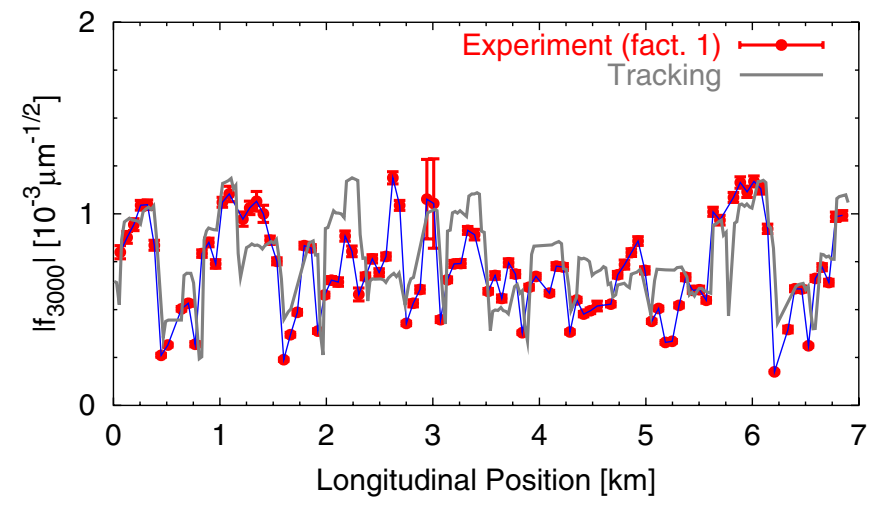

FIG. 9. (Color) Amplitude of the generating function term $f_{3000}$ versus longitudinal position from experiment and tracking model for the baseline machine at $26 \mathrm{GeV}$. The blue line is used to connect the experimental points.

$$
\sum_{n} k_{n} e^{i\left[(-j+k) \psi_{x n}+(m-l) \psi_{y n}\right]}
$$

where all the factors as beta functions and strengths are contained in $k_{n}$. Using these expressions, the change in the phase of the spectral line $(1-j+k, m-l)$ over a region free of nonlinear sources is computed. At the end of this region the betatron phases are simply propagated by $\Delta \psi_{x}$ and $\Delta \psi_{y}$, while the phases at the nonlinearities around the ring, $\psi_{x j}$ and $\psi_{y j}$, see the opposite transformation: $-\Delta \psi_{x}$ and $-\Delta \psi_{y}$, respectively. The changes in the phase of the spectral line are given by the sum of the change of the phase of the term $f_{j k l m}$ plus the change of the phase of the exponential part of Eq. (31), which yields

$$
\begin{aligned}
& (j-k) \Delta \psi_{x}+(l-m) \Delta \psi_{y}+(1-j+k) \Delta \psi_{x} \\
& \quad+(m-l) \Delta \psi_{y}=\Delta \psi_{x} .
\end{aligned}
$$

This means that the phase of all the spectral lines from the horizontal motion changes by the same amount, $\Delta \psi_{x}$, over a region free of nonlinear sources. This is apparent, in particular, for the horizontal tune line. Therefore, the following observable remains constant along sections free of nonlinear sources for any $m$ and $n$,

$$
\phi(m, n)-\phi(1,0)
$$

where $\phi(m, n)$ represents the phase of the spectral line with frequency $m Q_{x}+n Q_{y}$. As expected from Eq. (29), this phase observable changes abruptly at the location of the

TABLE I. Measured beam conditions for the experiments in 2002.

\begin{tabular}{lcc}
\hline \hline Energy [GeV] & 26 & 80 \\
Intensity [number of protons] & {$[0.5,6] \times 10^{10}$} & $3.5 \times 10^{10}$ \\
Number of bunches & 1 & 1 \\
Nominal tunes $\left[Q_{x}, Q_{y}\right]$ & $26.18,26.22$ & $26.18,26.22$ \\
Chromaticities $\left[Q_{x}^{\prime}, Q_{y}^{\prime}\right]$ & $1.3,1.3$ & $1.0,1.0$ \\
\hline \hline
\end{tabular}




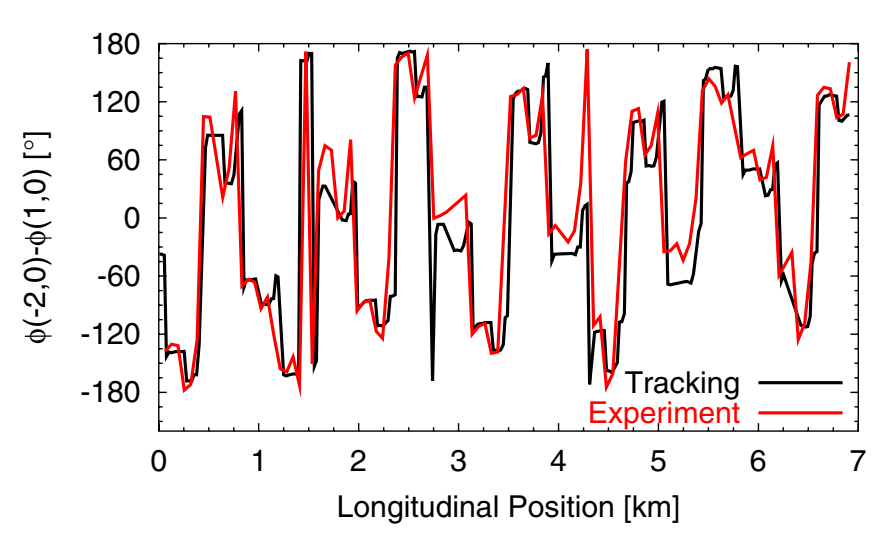

FIG. 10. (Color) Phase of the spectral line $(-2,0)$ minus the phase of the spectral line $(1,0)$ versus longitudinal position from experiment and tracking model for the baseline machine at $26 \mathrm{GeV}$.

nonlinear sources. The use of this phase observable in conjunction with the amplitude of the spectral line allows the unambiguous localization of nonlinear fields. In Fig. 10 this phase observable as measured from one single run is plotted together with the model prediction versus the longitudinal location for the baseline machine at $26 \mathrm{GeV}$. Because of the fact that experiment and model have different phase origins, a constant quantity had to be added to the experiment values to meet the model prediction.

\section{Linear coupling}

There are two resonances contributing to the linear coupling: $(1,-1)$ and $(1,1)$. The tunes of the experiments are much closer to the resonance $(1,-1)$ than to the $(1,1)$. For this reason the target of the experiments is the resonance $(1,-1)$. The first order resonance term driving this resonance is $h_{1001}$. The contribution of this term to the linearly normalized horizontal variable is given by Eq. (1):

$$
\begin{aligned}
\hat{x}(N)-i \hat{p}_{x}(N)= & \sqrt{2 I_{x}} e^{i\left(2 \pi \nu_{x} N+\psi_{x 0}\right)} \\
& -2 i f_{1001} \sqrt{2 I_{y}} e^{i\left(2 \pi \nu_{y} N+\psi_{y 0}\right)}, \\
\hat{y}(N)-i \hat{p}_{y}(N)= & \sqrt{2 I_{y}} e^{i\left(2 \pi \nu_{y} N+\psi_{y 0}\right)} \\
& -2 i f_{1001}^{*} \sqrt{2 I_{x}} e^{i\left(2 \pi \nu_{x} N+\psi_{x 0}\right)},
\end{aligned}
$$

where $f_{1001}^{*}$ is the complex conjugate of $f_{1001}$. The measurable amplitudes of the fundamental horizontal and vertical tunes are represented by line $(1,0)_{H}$ and line $(0,1)_{V}$, respectively. The measurable amplitude of the spectral line with the vertical tune in the horizontal plane is represented by line $(0,1)_{H}$ and corresponds, in the previous equations, to the quantity $\left|2 f_{1001} \sqrt{2 I_{y}}\right|$. Accordingly, the line $(1,0)_{V}$ corresponds to $\left|2 f_{1001} \sqrt{2 I_{x}}\right|$. To remove the dependency on the actions and on possible calibration constants the most robust observable related to of $\left|f_{1001}\right|$ is given by

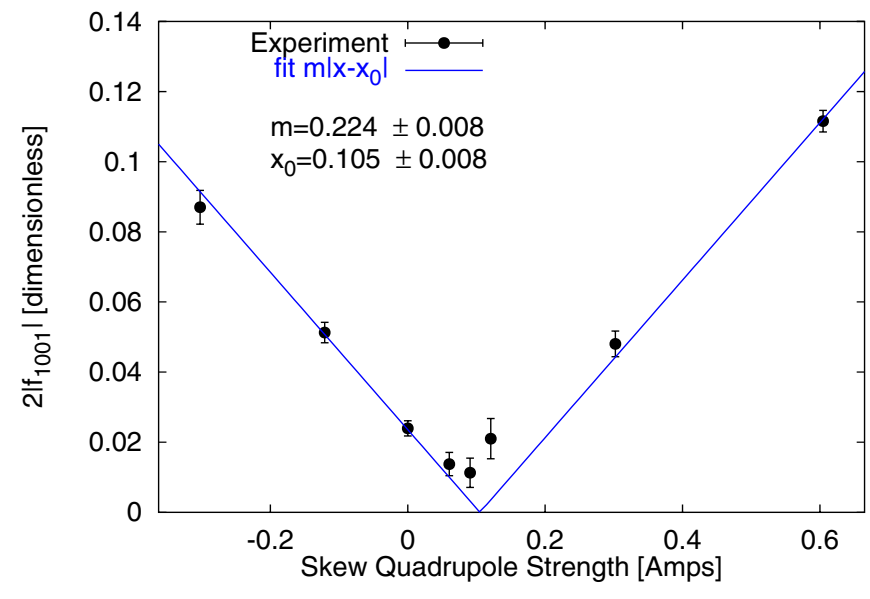

FIG. 11. (Color) Coupling resonance term versus the strength of skew quadrupoles.

$$
2\left|f_{1001}\right|=\sqrt{\frac{\operatorname{line}(0,1)_{H}}{\operatorname{line}(1,0)_{H}} \frac{\operatorname{line}(1,0)_{V}}{\operatorname{line}(0,1)_{V}}} .
$$

In Fig. 11 this new observable (average over all the BPMs) is plotted versus the strength of the skew quadrupoles together with a fit to the formula $m\left|x-x_{0}\right|$. The optimum skew setting is found via fitting the data.

\section{Exciting sextupolar resonances}

\section{Polarity error}

The extraction sextupoles were used to strongly excite sextupolar resonances at an energy of $120 \mathrm{GeV}$; see Table II for the beam conditions. In Fig. 12 the normalized amplitude of the spectral line $(-2,0)$ computed for all the BPMs for a $4 \sigma$ kick amplitude is plotted versus the longitudinal location. In the figure, the prediction from the nominal model is also shown and the location of the extraction sextupoles is represented by the dotted vertical lines. The agreement between model and experiment on average is good and the abrupt changes occur at the same places but the variations of the curves seem out of phase. This discrepancy could only be explained if the extraction sextupoles had opposite polarities than those of the chromaticity sextupoles. In Fig. 13 the same plot is shown but using the opposite polarities for all the extraction sextupoles. Hardware checks later confirmed that the polarities of these sextupoles were indeed reversed.

TABLE II. Measured beam conditions for the experiments during 2000.

\begin{tabular}{lcc}
\hline \hline Energy [GeV] & 26 & 120 \\
Intensity [number of protons] & $2 \times 10^{10}$ & $2 \times 10^{12}$ \\
Number of bunches & 1 & 84 \\
Nominal tunes $\left[Q_{x}, Q_{y}\right]$ & $26.62,26.58$ & $26.62,26.58$ \\
Chromaticities $\left[Q_{x}^{\prime}, Q_{y}^{\prime}\right]$ & $0.3,0.3$ & $0.52,0.16$ \\
\hline
\end{tabular}




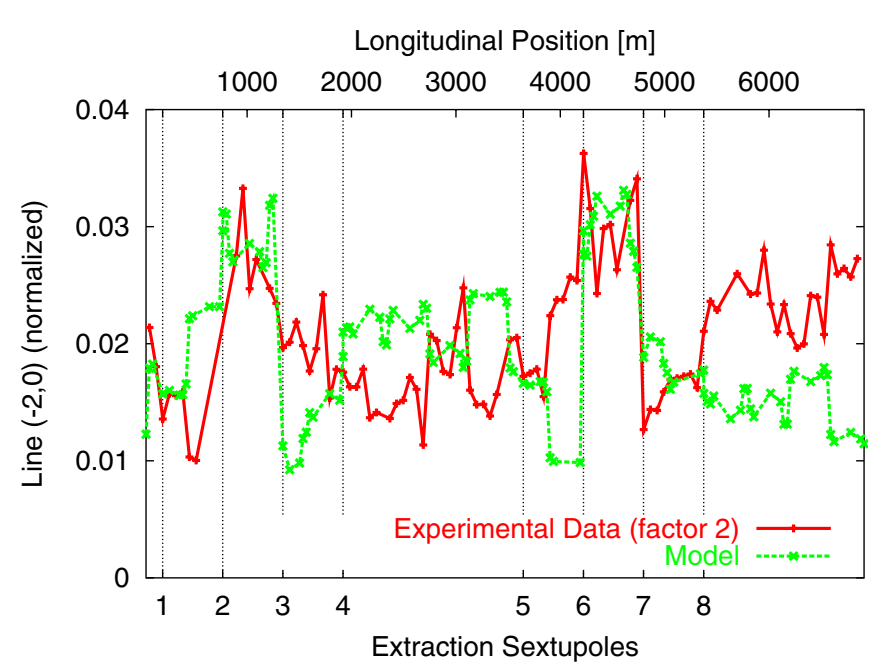

FIG. 12. (Color) Longitudinal variation of the spectral line $(-2,0)$ from experiment and from the nominal model.

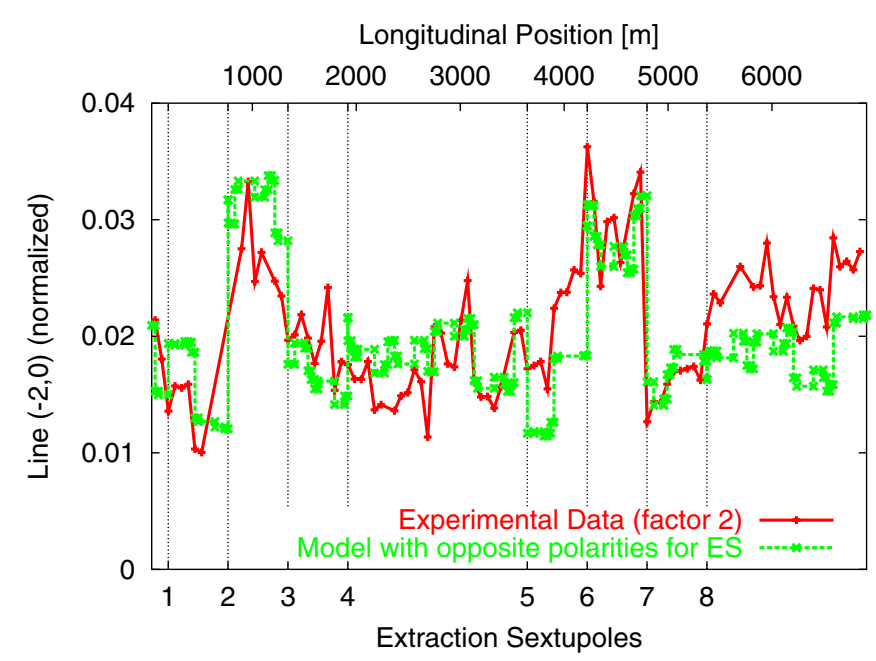

FIG. 13. (Color) Longitudinal variation of the spectral line $(-2,0)$ from experiment and model with opposite sextupole polarities.

\section{Effect of a missing extraction sextupole}

Measurements were also done with the extraction sextupoles powered to $(++++----) 100 \mathrm{~A}$ at $80 \mathrm{GeV}$, see Table I. In Fig. $14,\left|f_{3000}\right|$ is plotted versus the longitudinal position together with the results of the model. A factor of 2 is applied to the data in order to restore the effect of decoherence. The model and measurements show large discrepancies, in particular at the location of the first extraction sextupole. Whereas the model predicts a large change of the amplitude of this term, the measurement stays constant in that region. A possible explanation is that this particular extraction sextupole might not have been powered. Indeed, a comparison of the data with this new model without the first sextupole shows much better agreement (Fig. 15). Via the SPS alarm system [11], it was later confirmed that this sextupole was not powered.

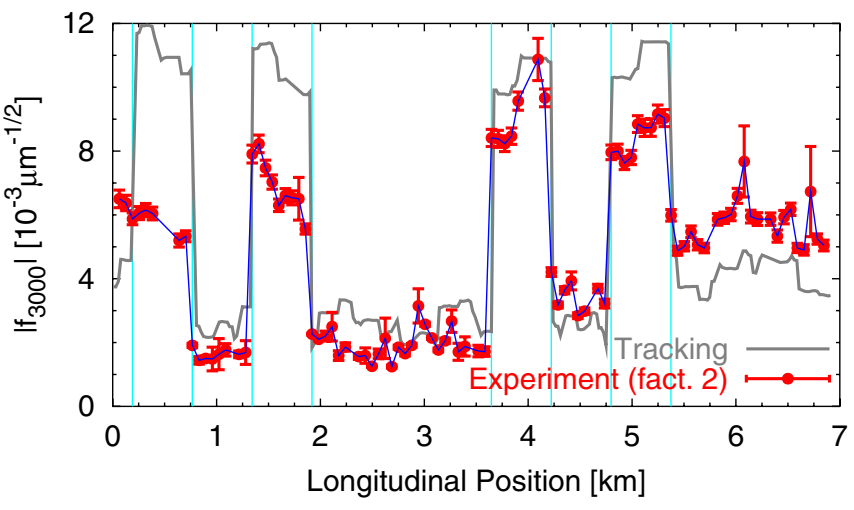

FIG. 14. (Color) Amplitude of the term $f_{3000}$ versus longitudinal position from experiment and tracking model for the SPS with extraction sextupoles powered to $(++++----) 100 \mathrm{~A}$ at $80 \mathrm{GeV}$. The blue line is used to connect the experimental points. The vertical lines denote the position of the extraction sextupoles.

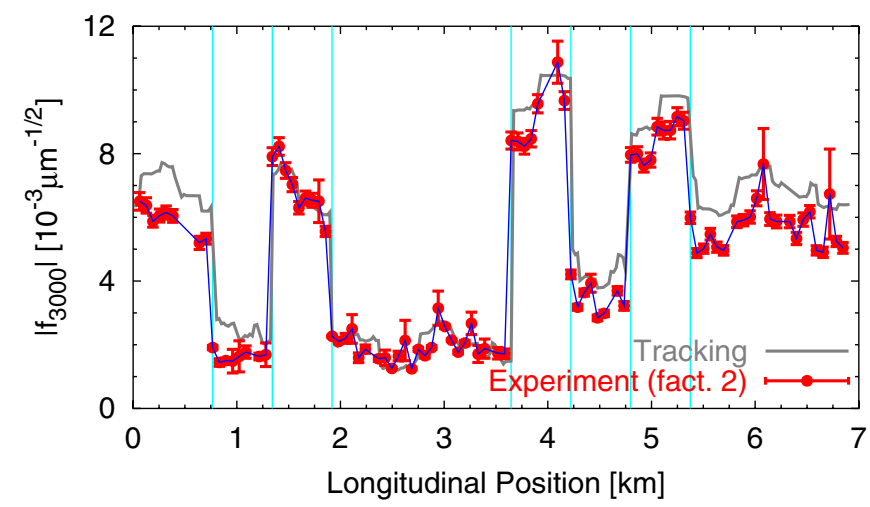

FIG. 15. (Color) Amplitude of the term $f_{3000}$ versus longitudinal position from experiment and tracking model for SPS with one sextupole disconnected $(+++----) 100 \mathrm{~A}$ at $80 \mathrm{GeV}$. The blue line is used to connect the experimental points. The vertical lines denote the position of the extraction sextupoles. Note that one sextupole with polarity " + " is missing.

\section{Effect of displaced sextupoles}

In another setup of the machine at $26 \mathrm{GeV}$, the first four extraction sextupoles were powered to $+30 \mathrm{~A}$ and the following four extraction sextupoles were powered to $-30 \mathrm{~A}$. The horizontal tune was moved to 26.69. The beam oscillations were damped due to decoherence, therefore the decoherence factor is applied to compare experiment and model. In Fig. 16 (top) the measured amplitude of the sextupolar resonance term $f_{3000}$ is plotted versus the longitudinal position together with results from the model. Apparently some ingredient is missing in the model which fails to explain the experimental data. In fact, if the closed orbit does not go through the center of a sextupole there is multipole feeddown and the particle experiences a quadrupolar field proportional to the offset. This quadrupolar field originates a beta beating, i.e., changes the nominal optic functions around the machine. This beta beating 

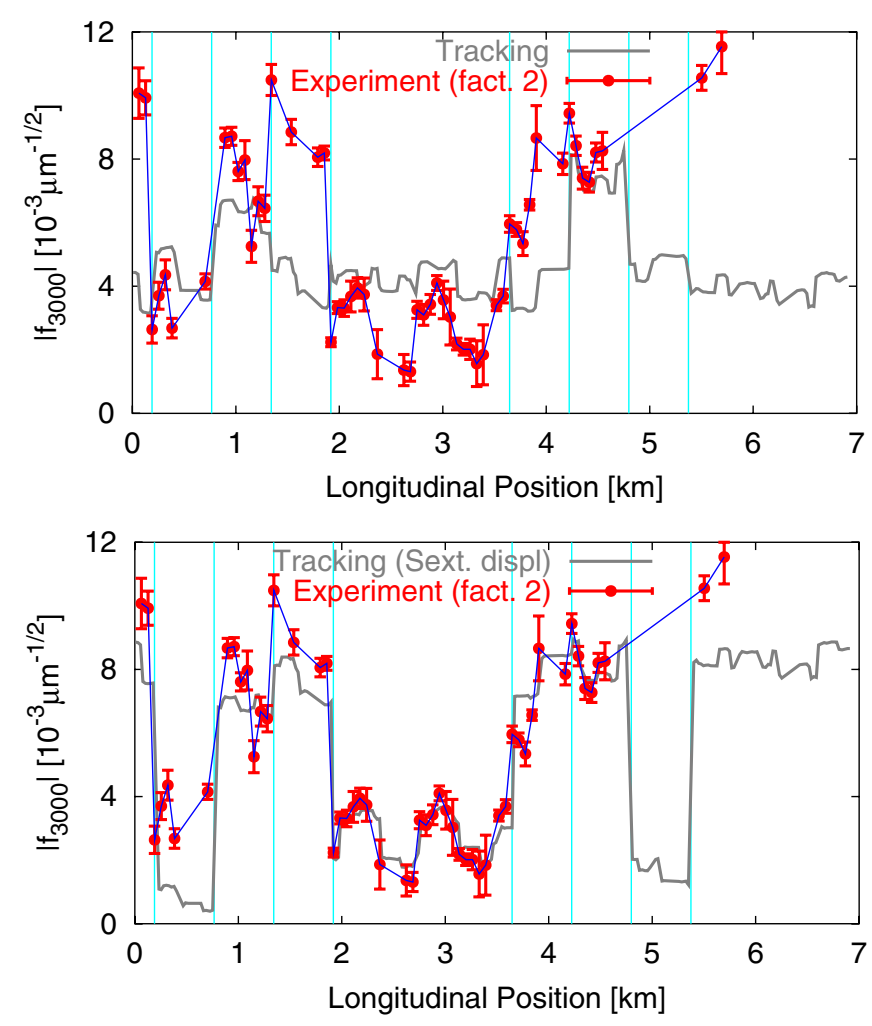

FIG. 16. (Color) Amplitude of the term $f_{3000}$ versus longitudinal position with extraction sextupoles on. Top: Experiment and nominal model with decoherence factor. Bottom: Experiment and model with displaced sextupoles and decoherence factor. The vertical lines show the position of the extraction sextupoles.

changes the strength of the resonances. For this reason, the displacements of the sextupoles with respect to the closed orbit were measured at the pickups and added to the model. This new model is used to compute the resonance term. The agreement between the experiment and the new model improved considerably as shown in Fig. 16 (bottom).

\section{EXPERIMENTS AT THE CERN PS BOOSTER SYNCHROTRON}

The CERN Proton Sychrotron Booster (PSB) is the first circular accelerator in the proton injector chain of the LHC and links the linear accelerator (LINAC2) with the proton synchrotron (PS). Apart from serving as a preinjector for the LHC, the PSB provides high intensity and high brightness beams for the ISOLDE physics facility and the CERN neutrinos to Gran Sasso experiment. The PSB consists of four parallel, superimposed rings, which are filled one after each other with $50 \mathrm{MeV}$ protons by means of a multiturn injection. The particles are then accelerated up to $1.4 \mathrm{GeV}$ and sent to the PS.

\section{A. Operation of the PS Booster}

The large incoherent space charge tune spreads [12] associated with the requested high intensity and high

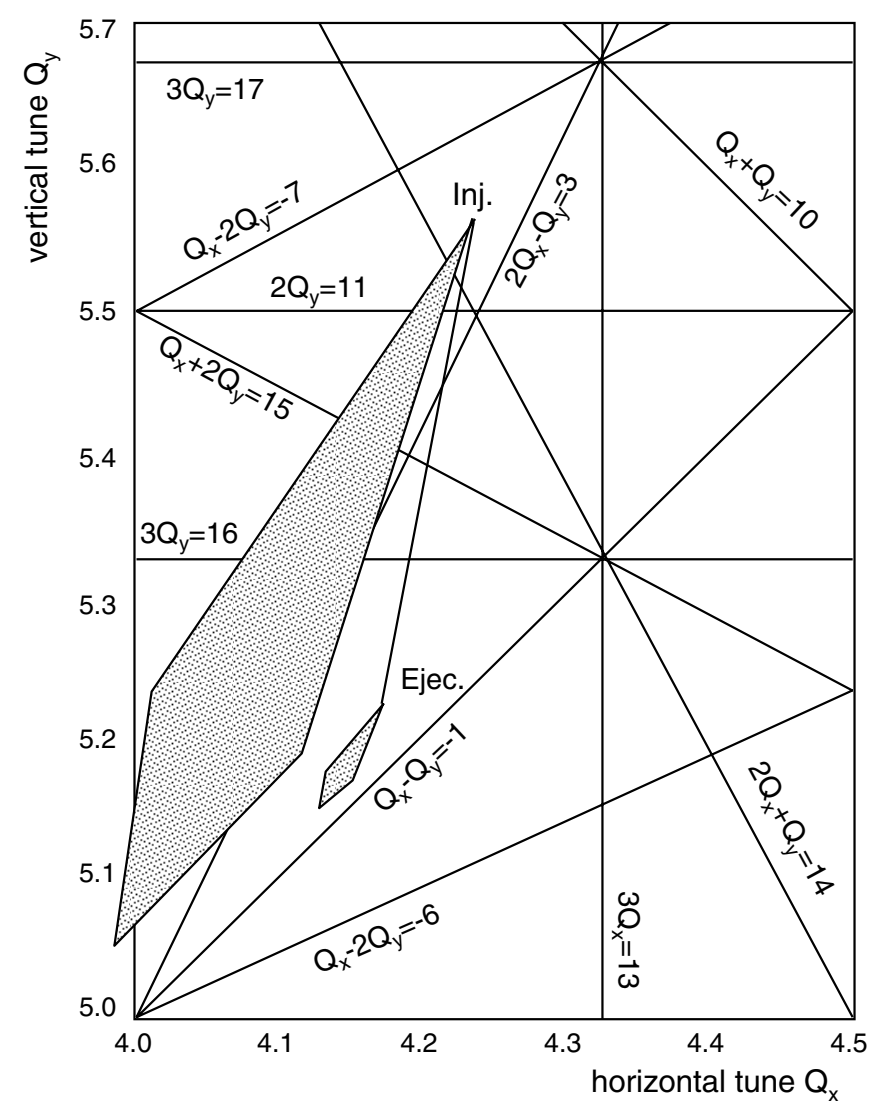

FIG. 17. PSB tune diagram for high intensity beams.

brightness beams, especially at injection, force a tune shift towards higher values to prevent particles from crossing the integer resonances $Q_{x}=4$ and $Q_{y}=5$. Figure 17 shows the tune diagram for the PSB with the resonance pattern up to third order. The large "necktie" shaped area in the diagram contains all the individual particles of the beam and covers several resonances. Since individual particles move within this area and repetitively cross the resonances, a detailed knowledge on strength and phase of resonance driving terms is mandatory in order to avoid beam blowup and subsequent beam losses. The tune spread shrinks proportionally to $1 / \beta \gamma^{2}$ as the beam is accelerated. The working point is lowered during acceleration towards the nominal values in an area clear of resonances (dynamic working point).

\section{B. Acquisition system}

The beam position signals from two BPMs are needed to construct the turn-by-turn complex signal. For this purpose, an acquisition system was installed to record transverse beam position over typically 1000 consecutive turns in the PSB.

Two of the standard closed orbit BPMs in ring 1 were equipped with new head amplifiers to increase the bandwidth, thus leading to proper beam position signals in both planes. The five electrode signals from each of the two 


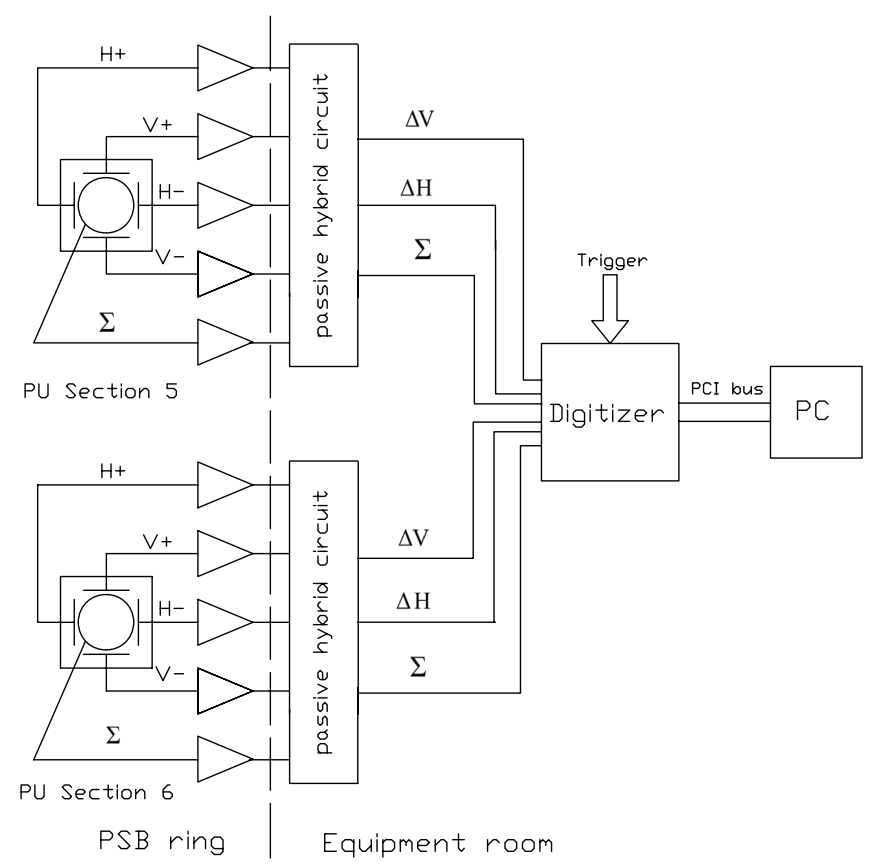

FIG. 18. Schematic view of the multiturn beam position acquisition system.

BPMs are amplified and passed onto a passive hybrid circuit to match the impedances and to build the horizontal and vertical delta signals. The signals are further transferred to the fast digitizer on separate channels. The digitizer simultaneously samples all selected input channels following a trigger coming from the control system. Data were recorded with two 8-bit 4 channel Acqiris digitizer modules [13]. The use of a memory extension of $2 \mathrm{MS} /$ channel enabled data taking over $4 \mathrm{~ms}$ at a sampling frequency of $500 \mathrm{MS} / \mathrm{s}$. Hence approximately 2500 turns could be stored at the revolution time $\tau_{\text {rev }} \approx 1.6 \mu \mathrm{s}$ at injection. Figure 18 gives an overview of the acquisition system. Table III summarizes the key data of the digitizer. The digitized signals are then converted into real beam position.

\section{Measurement setup}

High quality transverse beam position signals are the prerequisite for a successful resonance compensation. In principle, two basic measurement conditions are necessary for the analysis of the transverse particle motion: (i) The oscillation amplitude of the beam has to be reasonably large (some mm). (ii) The decoherence of the signal has to be avoided. The former is normally achieved with a kicker, the latter with chromaticity correction in both planes.

In the case of the PSB, no proper kicker is available, hence injection mis-steering (a deliberate deflection of the beam at injection) has to be used to excite the beam. As a consequence, only the first few thousand turns after injection into the machine could be analyzed, limiting the studies to injection energy $(50 \mathrm{MeV})$.

The decoherence of the beam position signals in both planes due to chromaticity is normally avoided by properly correcting the chromaticities with two independent sextupole families. The PSB is equipped with only one such a family, thus the signal decoherence in one of the transverse planes is unavoidable. The compensation in one plane leads to a degradation of the signal quality in the other plane. This is a further important restriction for the measurement of resonance driving terms, especially if coupling resonances are considered, where high signal quality in both planes is needed.

To obtain high quality signals, despite these limitations, the following adjustments on a special $50 \mathrm{MeV}$ flat machine cycle were done: (i) Only one-third of the ring was filled to obtain a quasibunched beam containing 1 to $2 \times$ $10^{11}$ protons. (ii) In order to conserve the bunch and consequently avoid longitudinal debunching, the RF was switched on prior to injection. (iii) Horizontal and vertical injection mis-steering were used to obtain sufficiently large oscillation amplitudes. (iv) The chromaticity was adjusted either to zero in one plane or to reasonably low values in both planes when coupling resonances were considered.

\section{Basic procedure to determine resonance driving terms}

With the above-mentioned setup for the PSB, the following procedure was applied to measure and compensate the bare machine resonance driving terms: (i) Each measurement was set up to sample one single resonance. To this end the tunes were set close to this specific resonance condition. The chromaticities were adjusted to best determine the corresponding resonance driving term. (ii) The normalized phase space was reconstructed from the measured beam positions using the general transfer matrix for

TABLE III. Key data of the digitizer.

\begin{tabular}{lcc}
\hline \hline Quantity & Value & Comment \\
\hline ADC & $8 \mathrm{bit}$ & \\
Sampling frequency & $500 \mathrm{MS} / \mathrm{s}$ & \\
Memory & $2 \mathrm{MS} / \mathrm{channel}$ & With extension \\
Maximum number of turns stored & 2500 & At injection energy \\
\hline \hline
\end{tabular}


TABLE IV. General relation between the vertical spectral lines and the amplitude and phase of the $h_{j k l m}$ Hamiltonian term, equivalent relations are found for the horizontal lines.

\begin{tabular}{lcc}
\hline \hline & Driving term & Vertical spectral line \\
\hline Line & & $(k-j, 1-l+m)$ \\
Amplitude & $\left|h_{j k l m}\right|$ & $2 I_{x}^{(j+k) / 2} 2 I_{y}^{(l+m-1) / 2} \frac{l \cdot\left|h_{j k l m}\right|}{\sin (|\hat{\phi}|)}$ \\
Phase & $\psi_{j k l m}$ & $\psi_{j k l m}-(j-k) \psi_{x_{0}}+(1-l+m) \psi_{y_{0}}-\frac{\pi}{2}+s g n(\hat{\phi})\left(\frac{\pi}{2}-|\hat{\phi}|\right)$ \\
& Where $\hat{\phi}=\pi\left[(j-k) \nu_{x}+(l-m) \nu_{y}\right.$ \\
\hline \hline
\end{tabular}

normalized coordinates,

$$
\left(\begin{array}{l}
Z_{2} \\
Z_{2}^{\prime}
\end{array}\right)=\left(\begin{array}{cc}
\cos (\Delta \mu) & \sin (\Delta \mu) \\
-\sin (\Delta \mu) & \cos (\Delta \mu)
\end{array}\right)\left(\begin{array}{l}
Z_{1} \\
Z_{1}^{\prime}
\end{array}\right)
$$

where $\Delta \mu$ is the phase advance between the two BPMs. (iii) SUSSIX [7] was used to determine the spectra of the motion in the normalized phase space. (iv) According to theory, the resonance strength and phase are calculated using the relations specified in Table IV. (v) Reference measurements with a defined multipole excitation were done to deduce the correct strength of the bare machine excitation and subsequently to find the proper compensation currents. (vi) With the knowledge of the strength $\left|h_{j k l m}\right|$ and the phase $\psi_{j k l m}$ of the resonance driving term, the compensation currents $I$ for two independent multipole families were calculated with

$$
\left(\begin{array}{l}
I_{1} \\
I_{2}
\end{array}\right)=M^{-1}\left(\begin{array}{l}
\left|h_{j k l m}\right| \cos \psi_{j k l m} \\
\left|h_{j k l m}\right| \sin \psi_{j k l m}
\end{array}\right)
$$

where $M$ represents a general matrix given by

$$
M=-\frac{1}{B \rho} \frac{1}{2^{n}} \frac{1}{n !}\left(\begin{array}{c}
n \\
l+m
\end{array}\right)\left(\begin{array}{c}
j+k \\
j
\end{array}\right)\left(\begin{array}{c}
l+m \\
l
\end{array}\right)\left(\begin{array}{cc}
\tilde{g}_{n(1)} l_{(1)} \beta_{x 1}^{(j+k) / 2} \beta_{y 1}^{(l+m) / 2} \cos \psi_{(1)} & \tilde{g}_{n(2)} l_{(2)} \beta_{x 2}^{(j+k) / 2} \beta_{y 2}^{(l+m) / 2} \cos \psi_{(2)} \\
\tilde{g}_{n(1)} l_{(1)} \beta_{x 1}^{(j+k) / 2} \beta_{y 1}^{(l+m) / 2} \sin \psi_{(1)} & \tilde{g}_{n(2)} l_{(2)} \beta_{x 2}^{(j+k) / 2} \beta_{y 2}^{(l+m) / 2} \sin \psi_{(2)}
\end{array}\right) .
$$

The suffixes (1) and (2) indicate the two compensation elements with the length $l$. The angles $\psi$ are the resonance phases of these elements with respect to the position of the BPM and $\tilde{g}_{n}$ represents the $n$th order gradient of the multipole magnets. The quantity $B \rho$ is the magnetic rigidity.

\section{E. Experimental Results \\ 1. Measurement program}

During standard machine operation, four resonances have to be compensated for a satisfactory performance of the PSB: (i) vertical second order resonance $2 Q_{y}=11$; (ii) vertical, systematic third order resonance $3 Q_{y}=16$ (the PSB rings consist of 16 identical sections.); (iii) third order coupling resonance $2 Q_{x}+Q_{y}=14$; (iv) third order coupling resonance $Q_{x}+2 Q_{y}=15$. The measurement program aimed at a compensation of the most relevant resonances $3 Q_{y}=16$ and $2 Q_{y}=11$ [14]. In addition, the linear coupling resonance $Q_{x}-Q_{y}=-1$ was analyzed in detail [15]. This resonance is not compensated in normal operation. Instead, it is deliberately excited during the multiturn injection to avoid beam losses. Furthermore, the driving terms of an alternative working point, i.e., the vertical tune is shifted down by one integer, were compared to the ones of the nominal tunes. The experimental results presented here refer to ring 1, which is one of the outer of the four PSB rings. Larger higher order multipole coefficients are expected in the outer rings, as the bending magnets are made from single yokes with four " $\mathrm{C}$ " type gaps. The compensation of the $3 Q_{y}=16$ resonance is discussed in detail, followed by a discussion of the results.

\section{The systematic 3rd order resonance $3 Q_{y}=16$}

For the determination of the corresponding resonance driving term $h_{0030}$, the vertical tune was adjusted close to

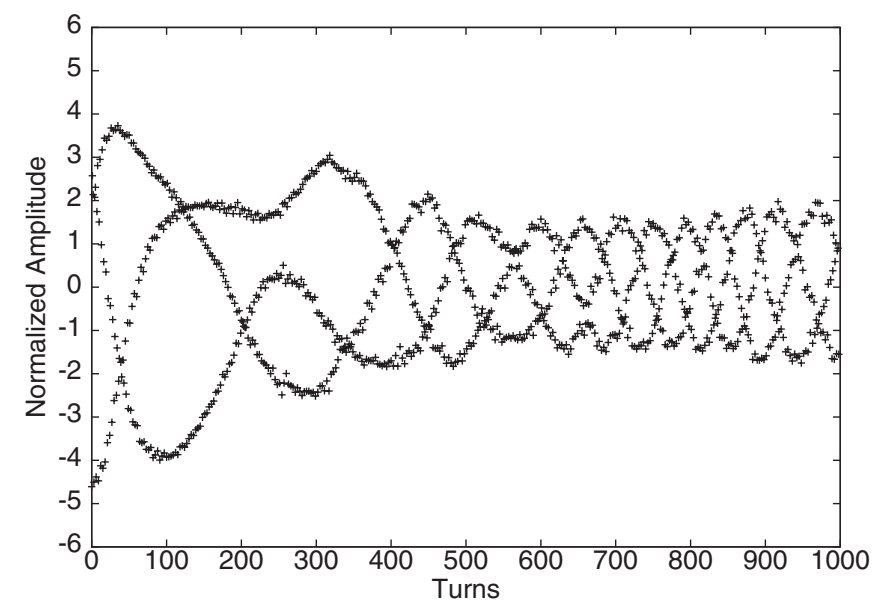

FIG. 19. Vertical beam position. 


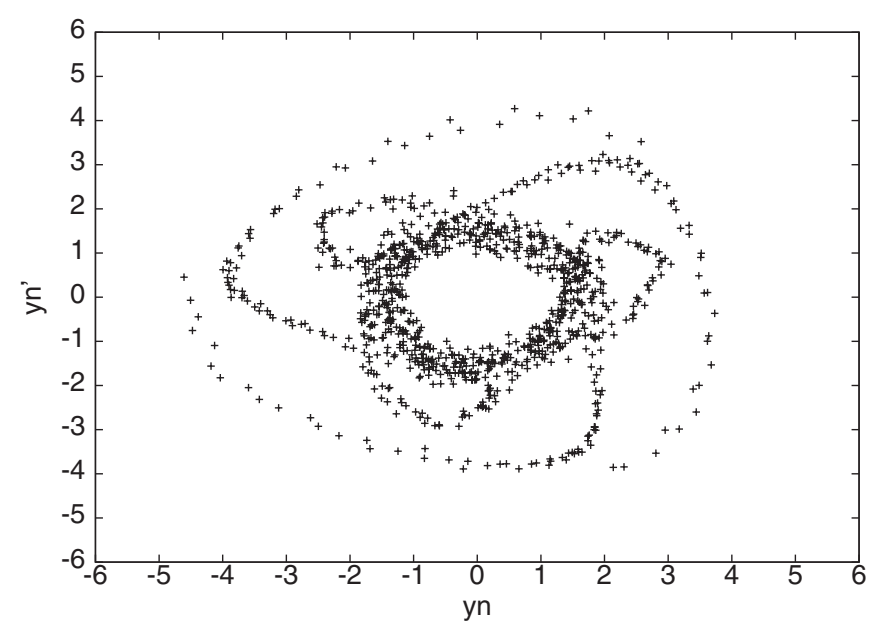

FIG. 20. Normalized vertical phase space without compensation.

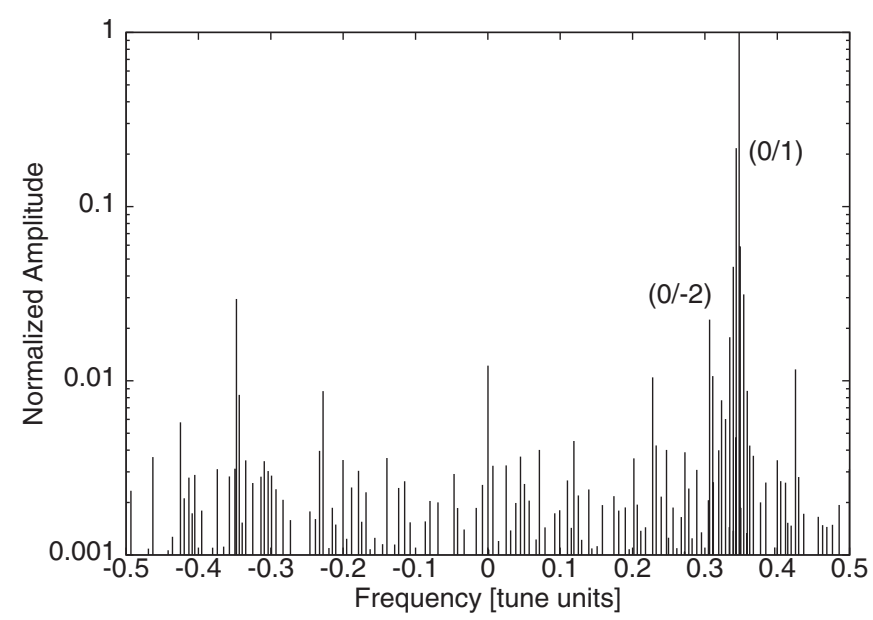

FIG. 21. Fourier spectrum without compensation.

the resonance condition, $Q_{y} \approx 5.345$ and the vertical chromaticity was corrected to zero. According to Table IV, the strength $\left|h_{0030}\right|$ and phase $\psi_{0030}$ are then expressed as

$$
\begin{gathered}
\left|h_{0030}\right|=\frac{1}{3} \frac{a_{y 1}}{a_{y 0}^{2}} \sin (|\hat{\phi}|), \\
\psi_{0030}=\phi_{y 1}+2 \psi_{y_{0}}+\frac{\pi}{2}-\operatorname{sgn}(\hat{\phi})\left(\frac{\pi}{2}-|\hat{\phi}|\right),
\end{gathered}
$$

where $a_{y 0}=\sqrt{2 I_{y}}$ is the amplitude of the tune line, $a_{y 1}$ and $\phi_{y 1}$ are the amplitude and the phase of the resonance spectral line $(0,-2)$, respectively.

(i) Measurement of the bare machine excitation.Figures 19 and 20 show the normalized vertical beam position and the corresponding phase space for a tune close to the third integer resonance condition over the first 1000 turns after injection. In the beginning the tune is slightly below the resonance, then the resonance is crossed after about 250 turns, and finally the tune is located a safe distance above the resonance. This is explained by fluctuations of the tune at injection into the PSB. The normalized phase space shows the typical triangular distortions when sextupoles are present. The phase swaps by $180^{\circ}$ at the moment of resonance crossing. In the vertical Fourier spectrum, Fig. 21, the $(0,-2)$ resonance line is labeled.

The strength and phase of the Hamiltonian term were calculated with Eqs. (40) and (41), the results are presented in Table V.

(ii) Reference measurements. - The skew sextupole XSK2L4 is located in a high $\beta_{y}$ region and therefore strongly excites the resonance. Tunes and chromaticity were adjusted as for the bare machine measurements. Table V compares the obtained resonance strength and phase to simulations. The strengths for the reference measurements and the simulation agree well, whereas the measured resonance phase is opposite to expectations, indicating an inversed polarity of the skew sextupole magnet. Taking into account the inversed polarity, measured and simulated resonance phase fit within ten degrees.

Furthermore, one notes that the strength of the bare machine Hamiltonian term is comparable to a strong excitation of the XSK2L4 sextupole which emphasizes that a compensation of this resonance is mandatory for a satisfactory performance of the PSB.

(iii) Compensation of the resonance. - With the knowledge of the bare machine excitation, the phases of the correction elements, and the calibration factor, the compensation currents for two independent skew sextupoles are obtained with Eqs. (38) and (39). The skew sextupoles XSK2L4 and XSK9L1 are suitable to compensate the resonance and were powered with the calculated currents, $I_{\mathrm{XSK} 2 \mathrm{~L} 4}=-29.3 \mathrm{~A}$ and $I_{\mathrm{XSK} 9 \mathrm{~L} 1}=+15.3 \mathrm{~A}$, respectively.

Figures 22 and 23 demonstrate the successful application of the resonance compensation. The normalized phase space appears "round" and is quasi-free from perturbing higher order terms. In the Fourier spectra the $(0,-2)$ spectral line has disappeared.

TABLE V. Overview on resonance strengths and phases for the bare machine and in case of excitation.

\begin{tabular}{lccc}
\hline \hline & Bare machine & Reference measurements & Simulation \\
\hline$\left|h_{0030}\right|\left[\mathrm{mm}^{-1 / 2}\right]$ & $9.0 \pm 0.6 \times 10^{-3}$ & $15.2 \pm 1.0 \times 10^{-3}$ & $14.3 \times 10^{-3}$ \\
$\psi_{0030}$ & $-21.4^{\circ} \pm 13.9^{\circ}$ & $157.2^{\circ} \pm 6.7^{\circ}$ & $347.2^{\circ}$ \\
\hline \hline
\end{tabular}




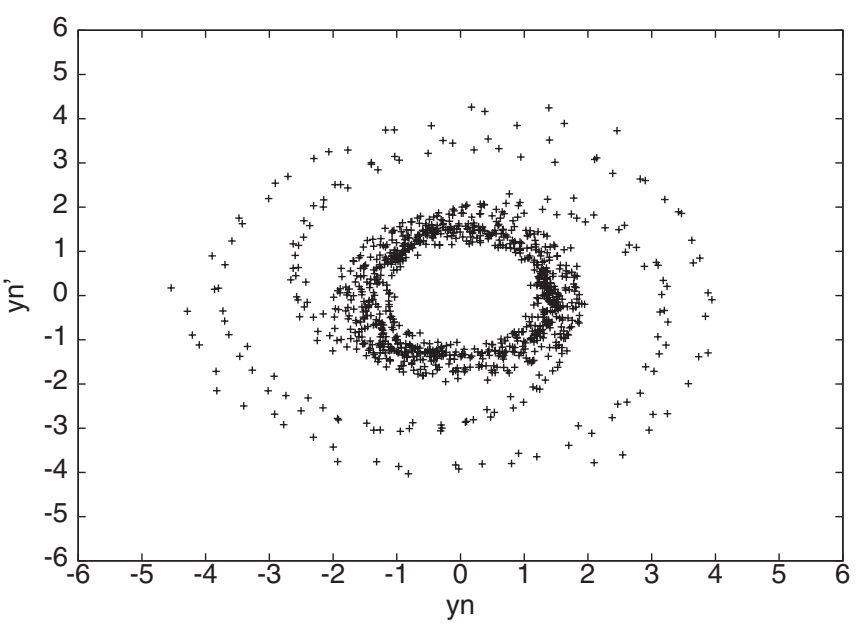

FIG. 22. Normalized vertical phase space after compensation.

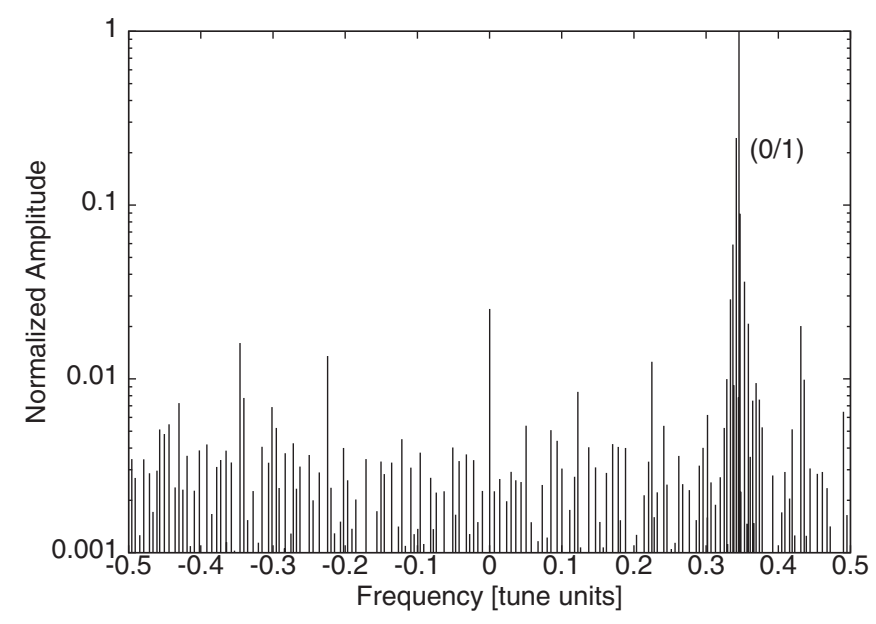

FIG. 23. Fourier spectrum after compensation.

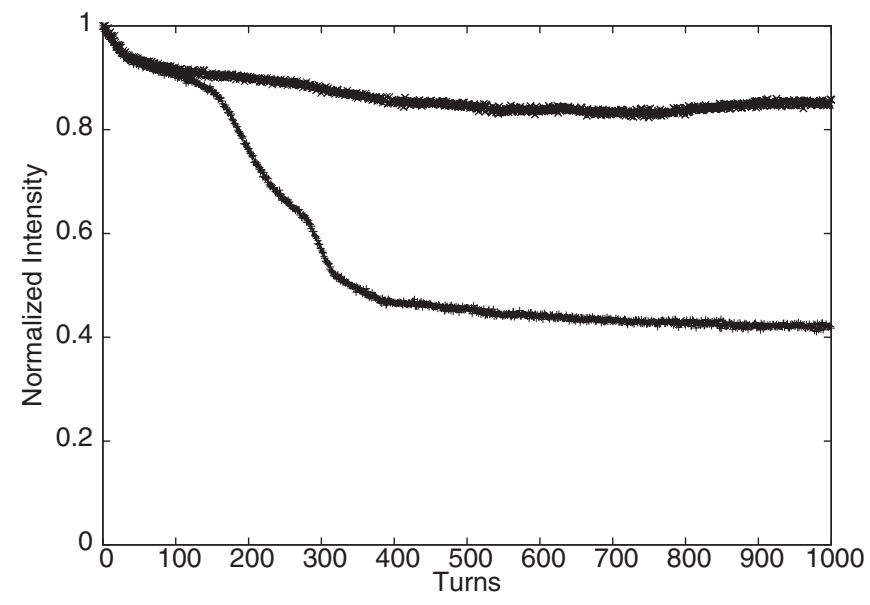

FIG. 24. Beam Intensity before and after compensation.
Figure 24 compares the beam intensities for the machine with and without resonance compensation. About $60 \%$ of the particles are lost within the first 300 turns in close vicinity to the resonance if no compensation is applied, otherwise the losses are small.

\section{Normal quadrupole resonance $2 Q_{y}=11$}

Figures 25-28 represent the situation for a tune close to the resonance condition without compensation. Large losses occur in the vicinity of the resonance and the normalized phase space has the characteristic elliptical shape due to the quadrupole perturbations of the bare machine. This resonance has to be compensated in standard machine operation.

During these measurements, the chromaticity was not corrected and the phase advance between the BPMs corresponding to the linear lattice was used for the analysis.

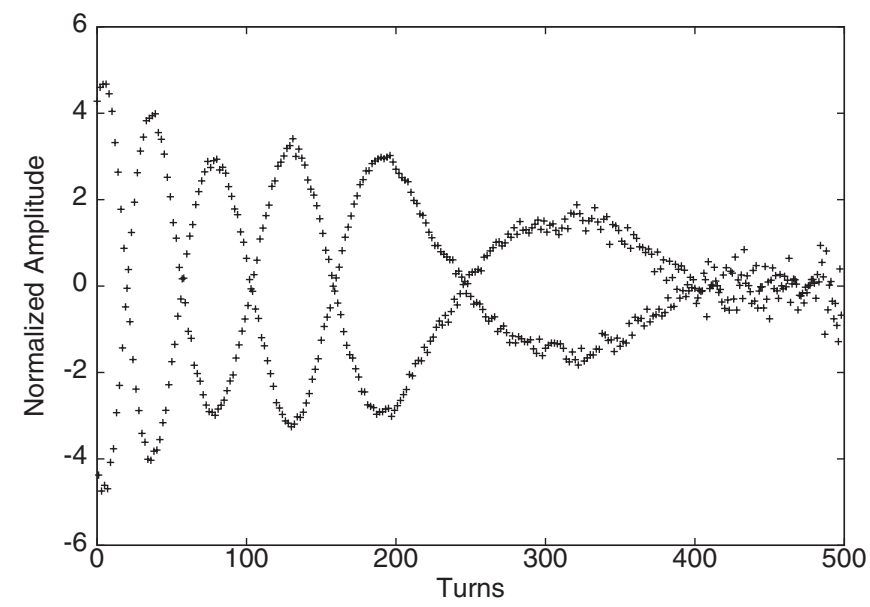

FIG. 25. Beam position.

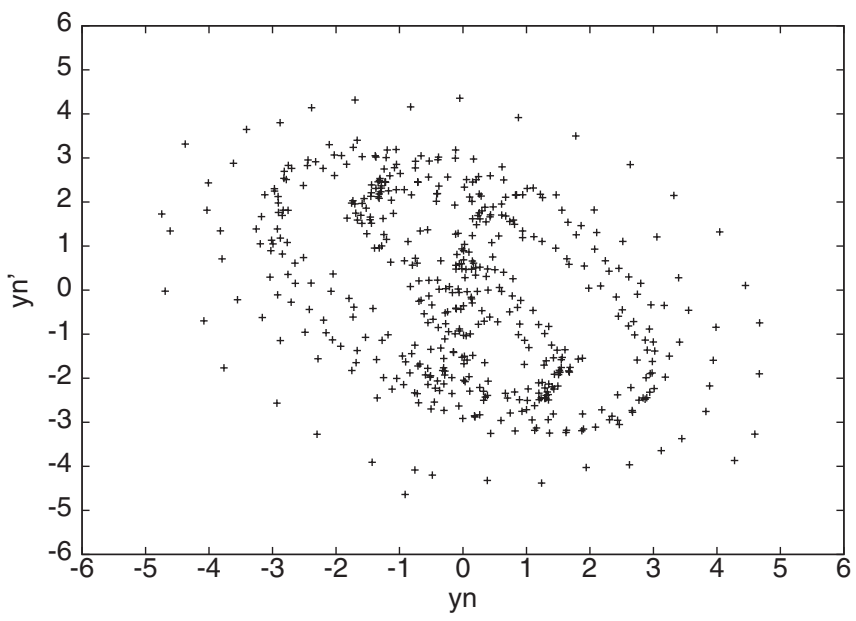

FIG. 26. Normalized vertical phase space without compensation. 


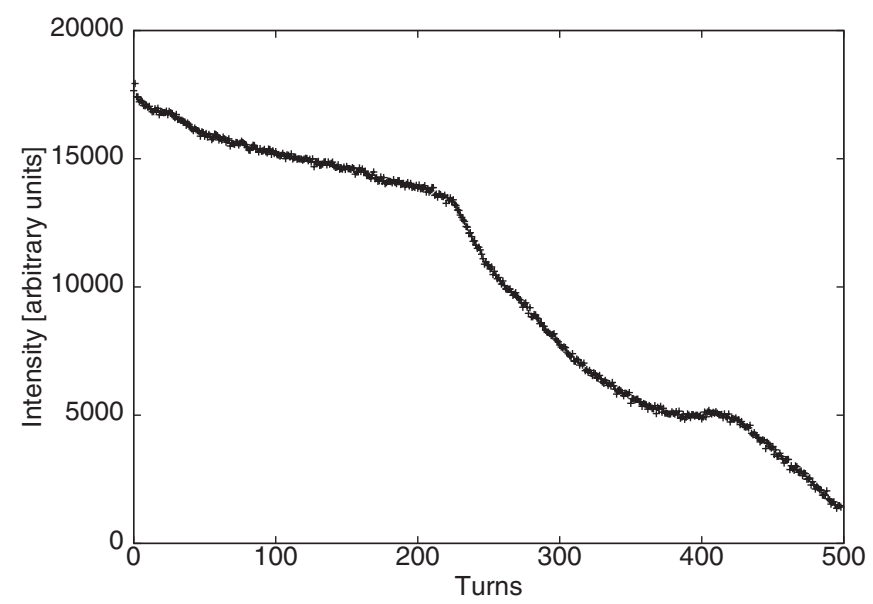

FIG. 27. Beam intensity without compensation.

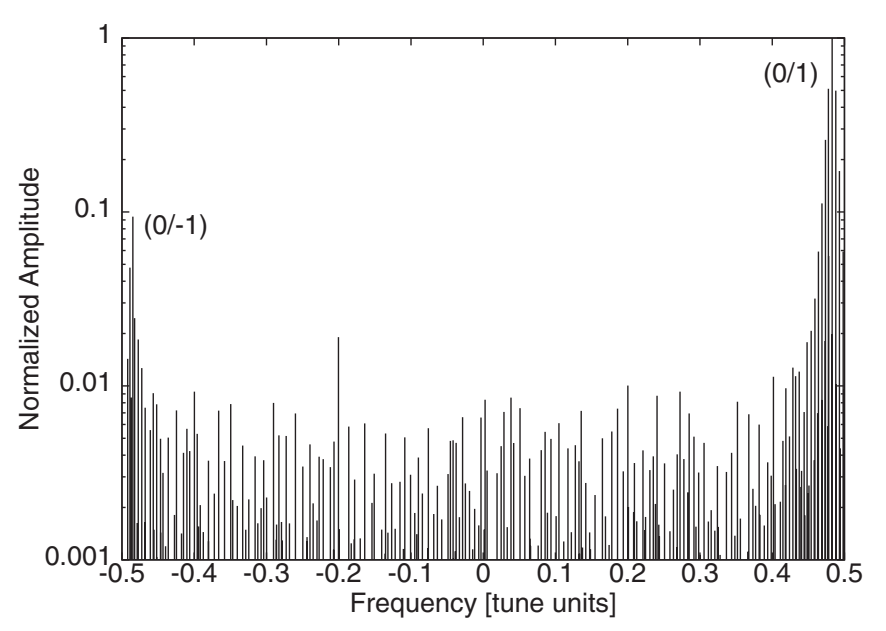

FIG. 28. Vertical Fourier spectrum without compensation.

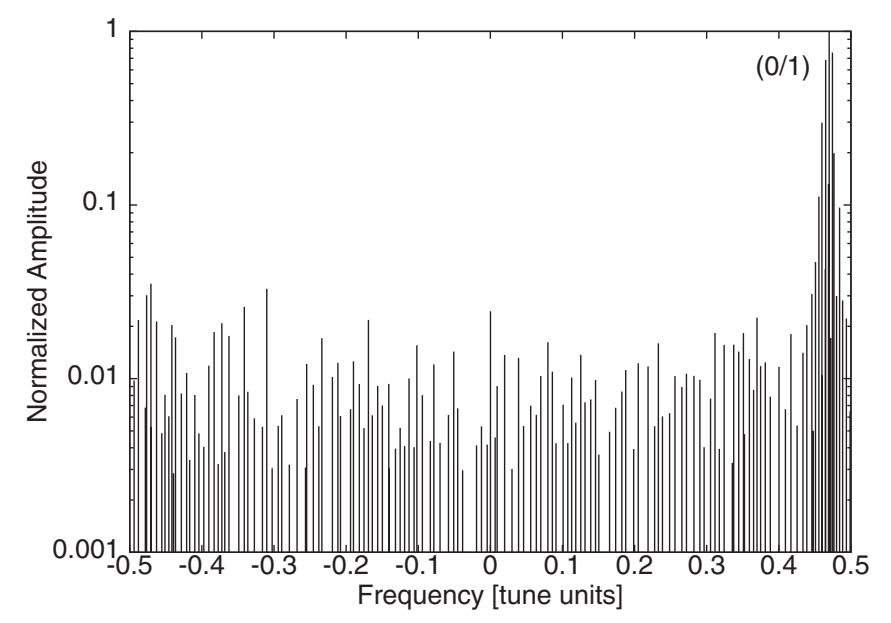

FIG. 29. Vertical Fourier spectrum with compensation.
The resonance strength and phase of the corresponding $h_{0020}$ driving term for the bare machine excitation were calculated to be

$$
\left|h_{0020}\right|=7.0 \pm 0.4 \times 10^{-3}, \quad \psi_{0020}=269.8^{\circ} \pm 8.2^{\circ},
$$

leading to the compensation currents:

$$
I_{\mathrm{QNO} 412 \mathrm{~L} 3}=+6.3 \mathrm{~A}, \quad I_{\mathrm{QNO} 816 \mathrm{~L} 3}=-2.8 \mathrm{~A} .
$$

Figure 29 shows that the resonance line disappears from the spectrum after the compensation.

\section{Linear coupling}

The linear coupling results in the difference and sum resonances $(1, \pm 1)$. The difference resonance $Q_{x}-Q_{y}=$ -1 has been studied because the tunes are closer to this resonance. Figures 30 and 31 show the horizontal and vertical Fourier spectra for the bare machine.

The spectral lines marked with $(0,1)$ in the horizontal and $(1,0)$ in the vertical spectrum correspond to the $h_{1001}$ driving term and therefore proof the strong excitation of

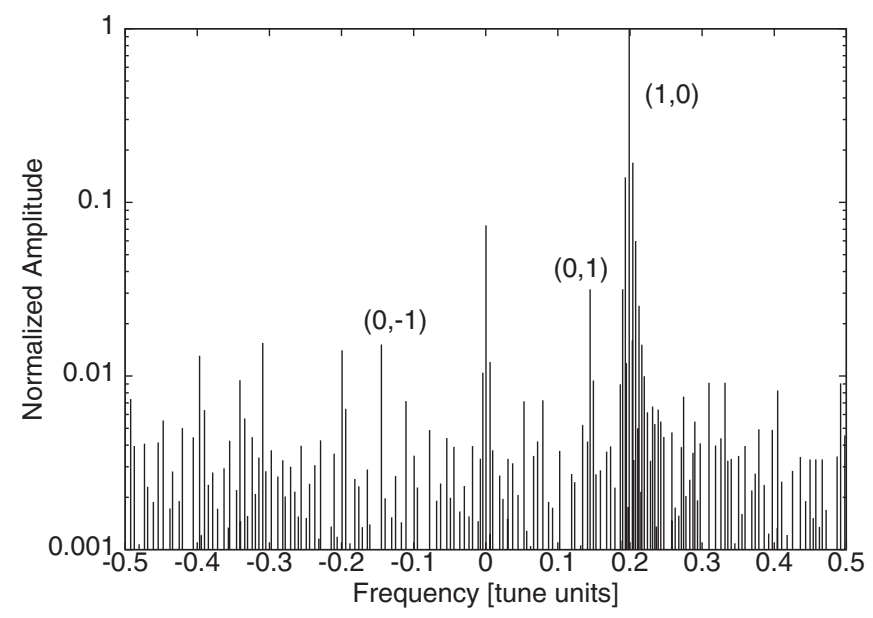

FIG. 30. Horizontal Fourier spectrum for bare machine.

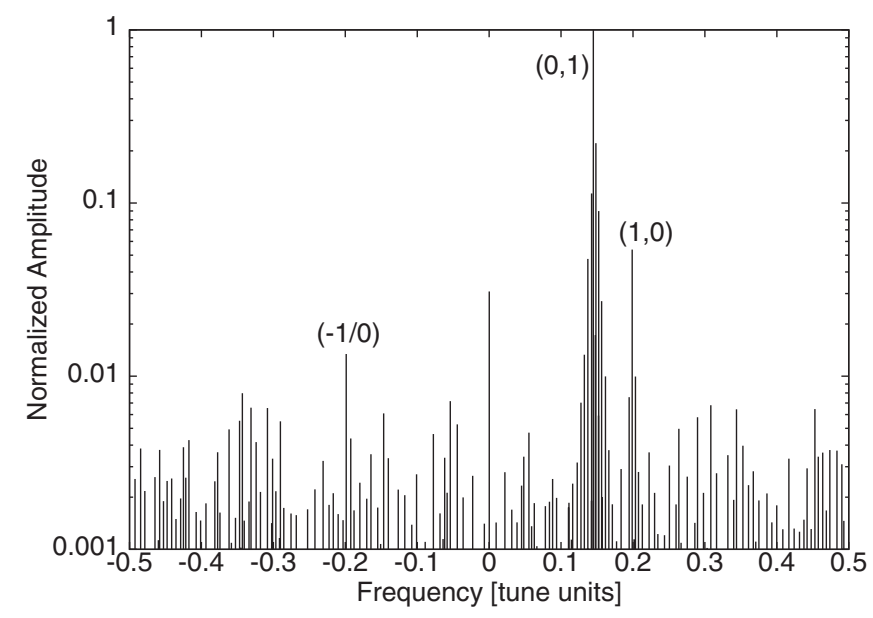

FIG. 31. Vertical Fourier spectrum for bare machine. 


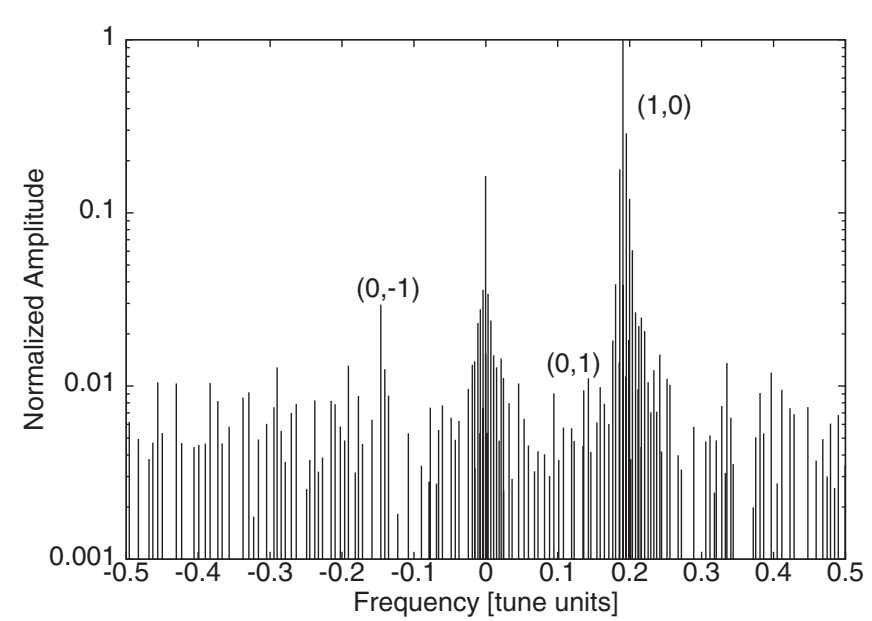

FIG. 32. Horizontal Fourier spectrum with compensation

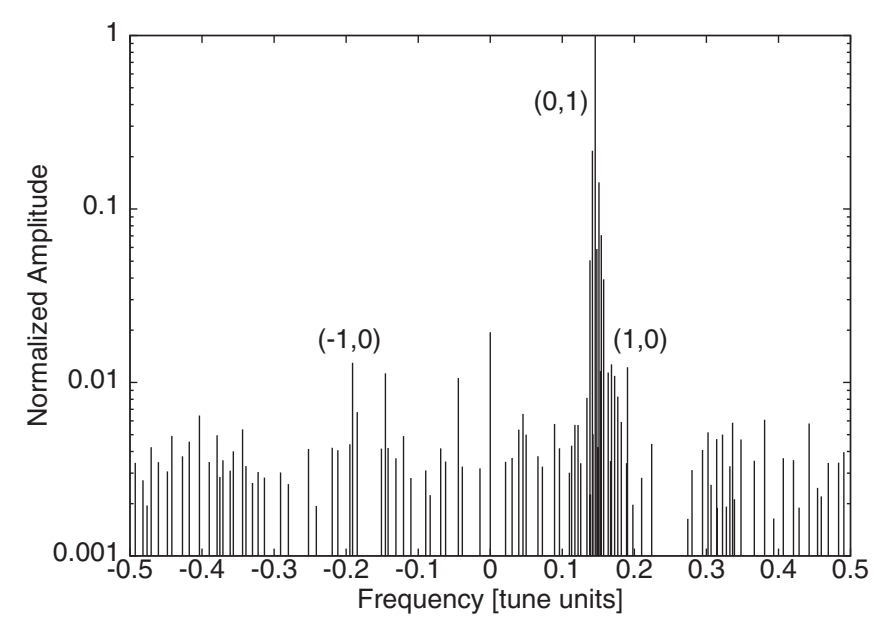

FIG. 33. Vertical Fourier spectrum with compensation.

the linear coupling difference resonance. The labeled lines $(0,-1)$ and $(-1,0)$ belong to the linear coupling sum resonance.

According to Eq. (36), both resonance spectral lines $(0,1)$ and $(1,0)$ are taken into account to obtain a solid observable. For the bare machine excitation the analysis of the spectra yields

$$
\left|h_{1001}\right|=7.1 \pm 0.1 \times 10^{-3}, \quad \psi_{1001}=282.8^{\circ} \pm 5.2^{\circ} \text {. }
$$

The compensation currents were calculated to

$$
I_{\mathrm{QSK} 210 \mathrm{~L} 3}=+3.6 \mathrm{~A}, \quad I_{\mathrm{QSK} 614 \mathrm{~L} 3}=+1.2 \mathrm{~A} .
$$

The resonance lines nearly disappeared in the background noise (Figs. 32 and 33), when these currents were applied.

\section{Alternative working point}

A new, alternative working point for the PSB was tested to avoid the $3 Q_{y}=16$ systematic resonance and hereby potentially increase the beam intensity and brightness. The
TABLE VI. Comparison of the bare machine resonance excitation for the old and new working point.

\begin{tabular}{lcc}
\hline \hline Hamiltonian term & Old WP & New WP \\
\hline$\left|h_{0020}\right|$ & $7.0 \pm 0.4 \times 10^{-3}$ & $2.9 \pm 0.2 \times 10^{-3}$ \\
$\left|h_{0030}\right|\left[\mathrm{mm}^{-1 / 2}\right]$ & $9.0 \pm 0.6 \times 10^{-3}$ & $2.2 \pm 0.4 \times 10^{-3}$ \\
\hline \hline
\end{tabular}

vertical tune was shifted one integer down to 4.23 , thus only nonsystematic resonances are present in the working area. Measurements of the bare machine resonance strengths $\left(\left|h_{0020}\right|\right.$ and $\left.\left|h_{0030}\right|\right)$ for the most important resonances $\left(2 Q_{y}=9\right.$ and $\left.3 Q_{y}=13\right)$ were performed and compared to the results of the former working point. Table VI summarizes the bare machine excitation for the old and new working point.

Since these resonances are smaller by a factor 3-4 for the new working point compared to the operational one the decision was taken to operate the PSB at the new working point as of 2005 .

\section{CONCLUSIONS}

This paper reports on the progress in the theoretical understanding of how resonance driving terms vary around the accelerator and how the theory has to be modified to take particle distributions into account rather than just a single particle. These theoretical advances allowed one to study various aspects of the theory in a controlled experiment at the SPS where the nonlinearities had to be introduced by some strongly powered sextupoles. In particular, this technique allowed one to detect the wrong polarity of the sextupole powering and to find missing sextupoles. The driving term studies at the PS Booster have been in the spirit of actually improving the performance of this machine. Despite the limits of the experimental setup it was possible to measure and correct the linear coupling and the skew sextupole resonances. Moreover, these studies allowed one to refine the integer parts of the tunes of this machine. One can therefore conclude that the resonance driving term technique has been matured long enough in the academic field to be applied for the performance enhancements of all sorts of accelerators.

\section{ACKNOWLEDGMENTS}

We thank F. Ruggiero, J. Gareyte, O. Brüning, and F. Zimmermann for the continuous monitoring of the SPS work and for useful discussions. Special thanks go to G. Rumolo and M. Hayes for providing simulation data and for collaborating in the SPS experiments. We thank: J. Klem and L. Jensen for help in developing the SPS software; R. Jones for the careful setting of the SPS BPM system; G. Arduini, F. Calderini, and N. Gonthier for their effort using the SPS alarm system; M. Albert, J. Wenninger, G. Arduini, K. Kornelis, and all the members of the SPS operation crew for their collaboration in SPS 
experiments. With regard to the PSB studies, we like to thank C. Carli, M. Chanel, and K. Schindl for numerous stimulating discussions concerning both the theory and the measurement of resonance driving terms. Furthermore, we would like to thank M. E. Angoletta for her help during the installation and configuration of the acquisition system. We are grateful for the assistance of J. Tan concerning the modifications of the PSB BPM electronics and the support of the PSB operations crew throughout the measurement campaign.

\section{APPENDIX A: SPECTRAL RESPONSE TO PARTICLE DISTRIBUTIONS}

\section{Centroid normalized horizontal coordinate}

To compute the centroid of a Gaussian beam as a function of the number of turns $N$, the expression of $h_{x}^{-}(N)$, given in Eq. (1), is integrated over the phase space weighted with the densities:

$$
\begin{gathered}
\rho_{x}\left(I_{x}, \psi_{x}\right)=\frac{1}{2 \pi} e^{-(1 / 2)\left(2 I_{x}+\bar{A}_{x}^{2}-2 \bar{A}_{x} \sqrt{2 I_{x}} \cos \psi_{x}\right)} \\
\rho_{y}\left(I_{y}, \psi_{y}\right)=\frac{1}{2 \pi} e^{-(1 / 2)\left(2 I_{y}+\bar{A}_{y}^{2}-2 \bar{A}_{y} \sqrt{2 I_{y}} \cos \psi_{y}\right)} \\
\rho_{s}\left(\delta, \psi_{s}\right)=\frac{\delta}{2 \pi \sigma_{s}^{2}} e^{-\delta^{2} /\left(2 \sigma_{s}^{2}\right)} .
\end{gathered}
$$

Note that the three densities describe Gaussian distributions but the longitudinal one uses a different phase-space variable. The horizontal coordinate of the centroid is therefore represented by

$$
\begin{aligned}
\overline{h_{x}^{-}}(N)= & \int_{0}^{\infty} d I_{x} d I_{y} d \delta \int_{0}^{2 \pi} d \psi_{x} d \psi_{y} d \psi_{s} \rho_{x}\left(I_{x}, \psi_{x}\right) \\
& \times \rho_{y}\left(I_{y}, \psi_{y}\right) \rho_{s}\left(\delta, \psi_{s}\right) h_{x}^{-}(N) .
\end{aligned}
$$

Since $h_{x}^{-}(N)$ contains a summation, this equation becomes a summation of integrals:

$$
\begin{aligned}
\overline{h_{x}^{-}}(N)= & L_{1100}(N) \\
& -2 i \sum_{j k l m} j f_{j k l m} L_{(j+k-1)(1-j+k)(l+m)(m-l)}(N),
\end{aligned}
$$

where $L_{n m l k}$ are defined as

$$
\begin{aligned}
L_{n m l k}= & \int d V^{6} \rho\left(I_{x}, \psi_{x}, I_{y}, \psi_{y}, \delta, \psi_{s}\right)\left(2 I_{x}\right)^{n / 2}\left(2 I_{y}\right)^{l / 2} \\
& \times e^{i\left[m\left(2 \pi \hat{\nu}_{x} N+\psi_{x}\right)+k\left(2 \pi \hat{\nu}_{y} N+\psi_{y}\right)\right]}
\end{aligned}
$$

for any integer $n, \quad m, \quad l, k, \quad d V^{6}$ stands for $d I_{x} d I_{y} d \delta d \psi_{x} d \psi_{y} d \psi_{s}$ and the tunes $\hat{\nu_{x}}$ and $\hat{\nu_{y}}$ with amplitude detuning and chromaticity were introduced in Eqs. (2) and (3). The following simplifications may be performed on the term $L_{n m l k}$.

Integral over the longitudinal plane.-Isolating all terms containing $\psi_{s}$, one gets

$$
\begin{aligned}
& \frac{1}{2 \pi} \int_{0}^{2 \pi} d \psi_{s} e^{i 2 \delta \xi_{m k} \cos \left(\pi Q_{s} N+\psi_{s}\right) \sin \left(\pi Q_{s} N\right) / Q_{s}} \\
& \quad=J_{0}\left[2 \delta \xi_{m k} \sin \left(\pi Q_{s} N\right) / Q_{s}\right]
\end{aligned}
$$

with $\xi_{m k}=\left(m Q_{x}^{\prime}+k Q_{y}^{\prime}\right)$ and $J_{0}$ being the Bessel function of order 0 . Grouping all terms containing $\delta$ and integrating over this variable yields

$$
\begin{aligned}
& \frac{1}{\sigma_{s}^{2}} \int_{0}^{\infty} d \delta \delta J_{0}\left(2 \delta \xi_{m k} \sin \left(\pi Q_{s}\right) / Q_{s}\right) e^{-\delta^{2} /\left(2 \sigma_{s}^{2}\right)} \\
& \quad=e^{-2 \xi_{m k}^{2} \sigma_{s}^{2} \sin ^{2}\left(\pi Q_{s} N\right) / Q_{s}^{2}} .
\end{aligned}
$$

Integral over the phases in the transverse planes.Isolating all the terms containing $\psi_{x}$ or $\psi_{y}$, the integral over these variables stays:

$$
\begin{aligned}
& \frac{1}{(2 \pi)^{2}} \int_{0}^{2 \pi} d \psi_{x} d \psi_{y} e^{\bar{A}_{x} \sqrt{2 I_{x}} \cos \psi_{x}+\bar{A}_{y} \sqrt{2 I_{y}} \cos \psi_{y}+i\left(m \psi_{x}+k \psi_{y}\right)} \\
& \quad=\mathrm{I}_{m}\left(\bar{A}_{x} \sqrt{2 I_{x}}\right) \mathrm{I}_{k}\left(\bar{A}_{y} \sqrt{2 I_{y}}\right)
\end{aligned}
$$

where the $\mathrm{I}_{n}$ represents the modified Bessel function of order $n$.

Using these simplifications $L_{n m l k}$ is given by

$$
\begin{aligned}
L_{\text {nmlk }}(N)= & \int_{0}^{\infty} d I_{x} d I_{y}\left(2 I_{x}\right)^{n / 2}\left(2 I_{y}\right)^{l / 2} e^{-(1 / 2)\left(2 I_{x}+\bar{A}_{x}^{2}+2 I_{y}+\bar{A}_{y}^{2}\right)} \\
& \times \mathrm{I}_{m}\left(\bar{A}_{x} \sqrt{2 I_{x}}\right) \mathrm{I}_{k}\left(\bar{A}_{y} \sqrt{2 I_{y}}\right) \\
& \times e^{i 2 \pi\left(m \nu_{x}+k \nu_{y}\right) N-2 \gamma_{m k}^{2} \sin ^{2}\left(\pi Q_{s} N\right)}
\end{aligned}
$$

with $\gamma_{m k}=\xi_{m k} \sigma_{s} / Q_{s}$. Notice that $\nu_{x}$ and $\nu_{y}$ contain only the amplitude detuning as expressed in Eq. (2). In the following section the Fourier transform of this expression is computed.

\section{Fourier transformation of the centroid motion}

The Fourier transform of $\overline{h_{x}^{-}}(N)$ is represented by $H_{x}^{-}(w)$. Since the Fourier transformation is a linear operator each term of the rhs of Eq. (A5) gives a contribution to $H_{x}^{-}(w)$, this is expressed by

$$
H_{x}^{-}(w)=\int d N \overline{h_{x}^{-}}(N) e^{-i w N} \equiv H_{x \text { tune }}^{-}(w)+\sum_{j k l m} H_{j k l m}^{-}(w) .
$$


To compute this transformation in the general case, the following integral has to be solved for arbitrary integers $m$ and $k$ :

$$
\mathcal{F}_{m k}(w)=\int d N e^{i 2 \pi\left(m \nu_{x}+k \nu_{y}-w / 2 \pi\right) N-2 \gamma_{m k}^{2} \sin ^{2}\left(\pi Q_{s} N\right)},
$$

which using the properties of the Bessel functions yields

$$
\begin{aligned}
\mathcal{F}_{m k}(w)= & e^{-\gamma_{m k}^{2}} \sum_{q=-\infty}^{\infty} \mathrm{I}_{q}\left(\gamma_{m k}^{2}\right) \\
& \times \delta_{\text {Dirac }}\left(m \nu_{x}+k \nu_{y}-w / 2 \pi+q Q_{s}\right) .
\end{aligned}
$$

$H_{x t u n e}^{-}(w)$ and $H_{x j k l m}^{-}(w)$ are expressed as follows:

$$
\begin{aligned}
H_{x \text { tune }}^{-}(w)= & \int_{0}^{\infty} d I_{x} d I_{y} \sqrt{2 I_{x}} e^{-(1 / 2)\left(2 I_{x}+\bar{A}_{x}^{2}+2 I_{y}+\bar{A}_{y}^{2}\right)} \\
& \times \mathrm{I}_{1}\left(\bar{A}_{x} \sqrt{2 I_{x}}\right) \mathrm{I}_{0}\left(\bar{A}_{y} \sqrt{2 I_{y}}\right) \mathcal{F}_{10}(w)
\end{aligned}
$$

$$
\begin{aligned}
H_{x j k l m}^{-}(w)= & -2 i j f_{j k l m} \int_{0}^{\infty} d I_{x} d I_{y}\left(2 I_{x}\right)^{(j+k-1) / 2}\left(2 I_{y}\right)^{(l+k) / 2} \\
& \times e^{-(1 / 2)\left(2 I_{x}+\bar{A}_{x}^{2}+2 I_{y}+\bar{A}_{y}^{2}\right)} \\
& \times \mathrm{I}_{(1-j+k)}\left(\bar{A}_{x} \sqrt{2 I_{x}}\right) \mathrm{I}_{(m-l)}\left(\bar{A}_{y} \sqrt{2 I_{y}}\right) \\
& \times \mathcal{F}_{(1-j+k)(m-l)}(w) .
\end{aligned}
$$

Using the properties of the Dirac delta function contained in $\mathcal{F}_{m k}(w)$, the integral over $I_{x}$ is performed expressing the tunes as in Eq. (2). The zero value of the Dirac delta function of Eq. (A13) is

$$
\begin{aligned}
2 I_{x, m k q}\left(w, I_{y}\right)= & \frac{1}{m \nu_{x x}^{\prime}+k \nu_{y x}^{\prime}}\left[w / 2 \pi-q Q_{s}\right. \\
& \left.-m\left(Q_{x}+\nu_{x y} 2 I_{y}\right)-k\left(Q_{y}+\nu_{y y} 2 I_{y}\right)\right]
\end{aligned}
$$

Since $I_{x, m k q}$ is an amplitude it must be larger or equal to zero. It has to be imposed that $I_{x, m k q}$ is zero when the rhs of Eq. (A16) is smaller than zero. To better interpret Eqs. (A14) and (A15), the following functions are defined:

$$
\begin{aligned}
\mathcal{A}_{x t \text { tune }}^{-}(w)= & \frac{1}{\left|\nu_{x x}^{\prime}\right|} \int_{0}^{\infty} d I_{y} \sqrt{2 I_{x, 100}\left(w, I_{y}\right)} e^{-(1 / 2)\left[2 I_{x, 100}\left(w, I_{y}\right)+\bar{A}_{x}^{2}+2 I_{y}+\bar{A}_{y}^{2}\right]} \mathrm{I}_{1}\left[\bar{A}_{x} \sqrt{2 I_{x, 100}\left(w, I_{y}\right)}\right] \mathrm{I}_{0}\left(\bar{A}_{y} \sqrt{2 I_{y}}\right) \\
\mathcal{A}_{x j k l m}^{-}(w)= & \frac{-2 i j f_{j k l m}}{\left|(1-j+k) \nu_{x x}^{\prime}+(m-l) \nu_{y x}^{\prime}\right|} \int_{0}^{\infty} d I_{y}\left[2 I_{x,(1-j+k)(m-l) 0}\left(w, I_{y}\right)\right]^{(j+k-1) / 2}\left(2 I_{y}\right)^{(l+k) / 2} \\
& \times e^{-(1 / 2)\left[2 I_{x,(1-j+k)(m-l) 0}\left(w, I_{y}\right)+\bar{A}_{x}^{2}+2 I_{y}+\bar{A}_{y}^{2}\right]} \mathrm{I}_{(m-l)}\left(\bar{A}_{y} \sqrt{\left.2 I_{y}\right)} \mathrm{I}_{(1-j+k)}\left[\bar{A}_{x} \sqrt{2 I_{x,(1-j+k)(m-l) 0}\left(w, I_{y}\right)}\right],\right.
\end{aligned}
$$

where $I_{x, m k 0}\left(w, I_{y}\right)$ is the function defined in Eq. (A16) with $q=0$ ( $q$ is explicitly reintroduced later). Last, the terms of the Fourier transform $H_{x t u n e}^{-}(w)$ and $H_{x j k l m}^{-}(w)$ may be written as

$$
\begin{aligned}
H_{x \text { tune }}^{-}(w) & =\mathcal{A}_{x \text { tune }}^{-}(w) e^{-\gamma_{10}^{2}} \mathrm{I}_{0}\left(\gamma_{10}^{2}\right)+\sum_{q=1}^{\infty} e^{-\gamma_{10}^{2}} \mathrm{I}_{q}\left(\gamma_{10}^{2}\right)\left[\mathcal{A}_{x \text { tune }}^{-}\left(w+q 2 \pi Q_{s}\right)+\mathcal{A}_{x \text { tune }}^{-}\left(w-q 2 \pi Q_{s}\right)\right] \\
H_{x j k l m}^{-}(w)= & \mathcal{A}_{x j k l m}^{-}(w) e^{-\gamma_{(1-j+k)(m-l)}^{2}} \mathrm{I}_{0}\left(\gamma_{(1-j+k)(m-l)}^{2}\right)+\sum_{q=1}^{\infty} e^{-\gamma_{(1-j+k)(m-l)}^{2}} \mathrm{I}_{q}\left(\gamma_{(1-j+k)(m-l)}^{2}\right)\left[\mathcal{A}_{x j k l m}^{-}\left(w+q 2 \pi Q_{s}\right)\right. \\
& \left.+\mathcal{A}_{x j k l m}^{-}\left(w-q 2 \pi Q_{s}\right)\right] .
\end{aligned}
$$

The interpretation of the formula is quite simple. If the chromaticity is zero $\gamma_{m k}=0$ for all $m, k$. All the modified Bessel functions with an index $q>0$ vanish:

$$
H_{x}^{-}(w)=\mathcal{A}_{x \text { tune }}^{-}(w)+\sum_{j k l m} \mathcal{A}_{x j k l m}^{-}(w) .
$$

This equation shows how the spectral lines of the singleparticle motion equation (1) are transformed into distribution functions. The effect of chromaticity, as seen from Eqs. (A19) and (A20), leads to an infinite number of peaks around the principal ones displaced by integer multiples of $Q_{s}$, this integer represented by $q$ is the order of the sideband. The functions $\mathcal{A}_{x j k l m}^{-}(w)$ still contain the integral over $I_{y}$ which has to be solved numerically.

[1] J. Bengtsson, CERN 88-05, 1988.

[2] R. Bartolini and F. Schmidt, LHC Project Report 98; in Proceedings of the Workshop on "Nonlinear and 
Collective Phenomena in Beam Physics," Arcidosso, 1996, AIP Conference Proceedings 395 (AIP, New York, 1996).

[3] R. Bartolini and F. Schmidt, Part. Accel. 59, 93 (1998), http://wwwslap.cern.ch/frs/report/lines97.pdf.

[4] R. Bartolini, L. H. A. Leunissen, Y. Papaphilippou, F. Schmidt, and A. Verdier, CERN-SL-99-032 AP.

[5] G. Rumolo and R. Tomás, Nucl. Instrum. Methods Phys. Res., Sect. A 528, 670 (2004).

[6] F. Schmidt, CERN SL/94-56 (AP).

[7] R. Bartolini and F. Schmidt, CERN SL-Note-98-017 (AP).

[8] É. Forest, Beam Dynamics: A New Attitude and Framework (Harwood Academic, Chichester, 1998), pp. $168-171$.

[9] X. Altuna et al., CERN SL-MD Note 045, 1998.
[10] X. Altuna et al., CERN SL-Note-99-017 MD.

[11] G. Arduini, F. Calderini, and N. Gonthier (private communication).

[12] K. Schindl, CERN/PS 99-012 (DI).

[13] M.E. Angoletta and A.-S. Müller, CERN/PS 2002-030 (AE).

[14] P. Urschütz, Measurement and Compensation of Second and Third Order Resonances at the CERN PS Booster, EPAC 2004, Lucerne, Switzerland.

[15] M. Benedikt, C. Carli, M. Chanel, F. Schmidt, and P. Urschütz, Betatron Resonance Studies at the CERN PS Booster by Harmonic Analysis of Turn-by-Turn Beam Position Data, EPAC 2004, Lucerne, Switzerland. 